\title{
Quotients and subgroups of Baumslag-Solitar groups
}

\author{
Gilbert Levitt
}

\begin{abstract}
We determine all generalized Baumslag-Solitar groups (finitely generated groups acting on a tree with all stabilizers infinite cyclic) which are quotients of a given Baumslag-Solitar group $B S(m, n)$, and (when $B S(m, n)$ is not Hopfian) which of them also admit $B S(m, n)$ as a quotient. We determine for which values of $r, s$ one may embed $B S(r, s)$ into a given $B S(m, n)$, and we characterize finitely generated groups which embed into some $B S(n, n)$.
\end{abstract}

\section{Introduction and statement of results}

This paper studies various aspects of the Baumslag-Solitar groups $B S(m, n)=\langle a, t|$ $\left.t a^{m} t^{-1}=t^{n}\right\rangle$. These groups are HNN extensions of $\mathbb{Z}$, and they are best understood within the class of generalized Baumslag-Solitar (GBS) groups. These are fundamental groups of finite graphs of groups $\Gamma$ with all vertex and edge groups $\mathbb{Z}$ (equivalently, finitely generated groups $G$ acting on a tree with infinite cyclic edge and vertex stabilizers); they are represented by $\Gamma$, viewed as a graph labelled by indices of edge groups in vertex groups (see Section 2).

It is well-known [2, 5] that Baumslag-Solitar groups are not always Hopfian: they may be isomorphic to proper quotients. In fact, we will see that there exist infinitely many nonisomorphic GBS groups $G$ which are epi-equivalent to $B S(4,6)$ : there exist non-injective epimorphisms $B S(4,6) \rightarrow G \rightarrow B S(4,6)$.

A GBS group $G$ is a quotient of some $B S(m, n)$ if and only if it is 2-generated. The first main result of this paper is the determination of the values of $m, n$ for which a given $G$ is a quotient of $B S(m, n)$.

It follows from Grushko's theorem (see [13]) that the only possibilities for the topology of a labelled graph $\Gamma$ representing a non-cyclic 2-generated GBS group $G$ are: a segment, a circle, or a lollipop (see Figure 1, vertices are named $v_{i}, w_{i}$, labels are $q_{j}, r_{j}, x_{j}, y_{j}$; we view a circle as a lollipop with no arc attached). We define numbers $Q, R, X, Y$ as the products of the labels with the corresponding letter, with $Q=R=1$ if $\Gamma$ is a circle.

Theorem 1.1 (Theorem 4.1). Let $\Gamma$ be a labelled graph as on Figure 11 representing a 2-generated GBS group $G$ other than $\mathbb{Z}$ or the Klein bottle group $K$.

- Segment case: $G$ is a quotient of $B S(m, n)$ if and only if $m=n$ and $m$ is divisible by $Q$ or $R$;

- Circle/lollipop case: $G$ is a quotient of $B S(m, n)$ if and only if $(m, n)$ is an integral multiple of $(Q X, Q Y)$ or $(Q Y, Q X)$.

Using this, one may describe all GBS quotients of a given $B S(m, n)$. In particular (see Proposition [5.6), one may determine which $B S(m, n)$ have infinitely many GBS quotients up to isomorphism.

We also consider Baumslag-Solitar quotients of a given 2-generated GBS group $G$. This is interesting only for groups represented by a circle or a lollipop (a group represented by a segment has no Baumslag-Solitar quotient, except sometimes the Klein bottle group 


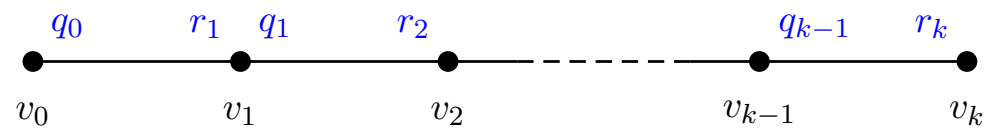

segment

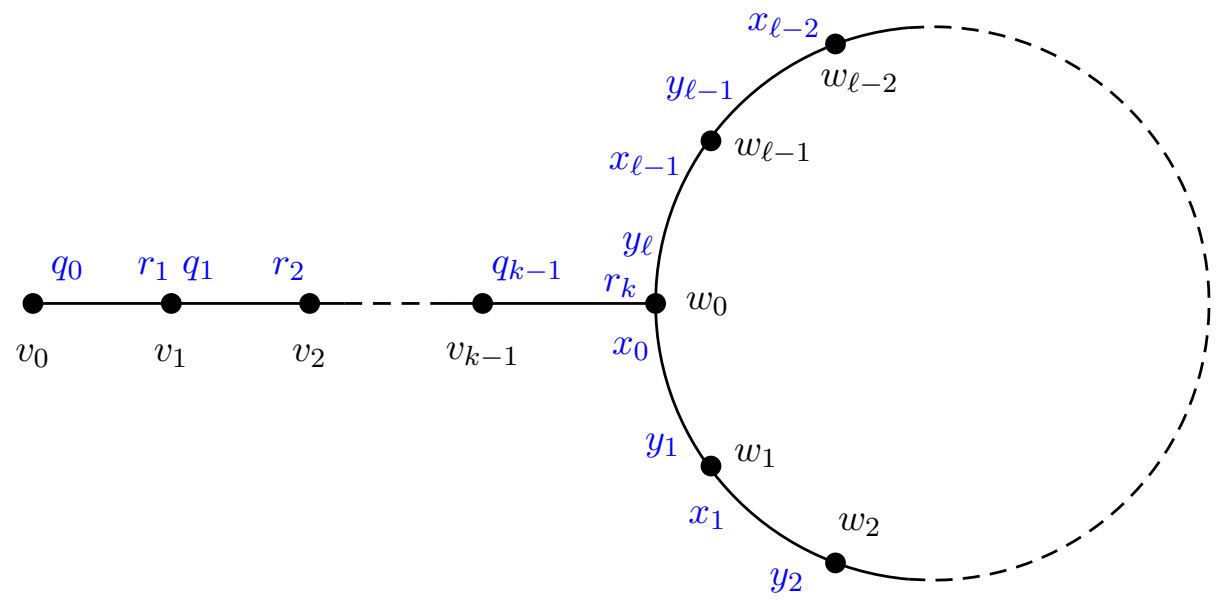

lollipop

Figure 1: representing 2-generated GBS groups

$K)$. By the previous theorem there is a "smallest" Baumslag-Solitar group mapping onto $G$, namely $B S(Q X, Q Y)$, and we determine whether $G$ maps onto $B S(Q X, Q Y)$. The general statement (Theorem 6.6) is a little technical, so we limit ourselves here to the groups pictured on Figure 2 ,

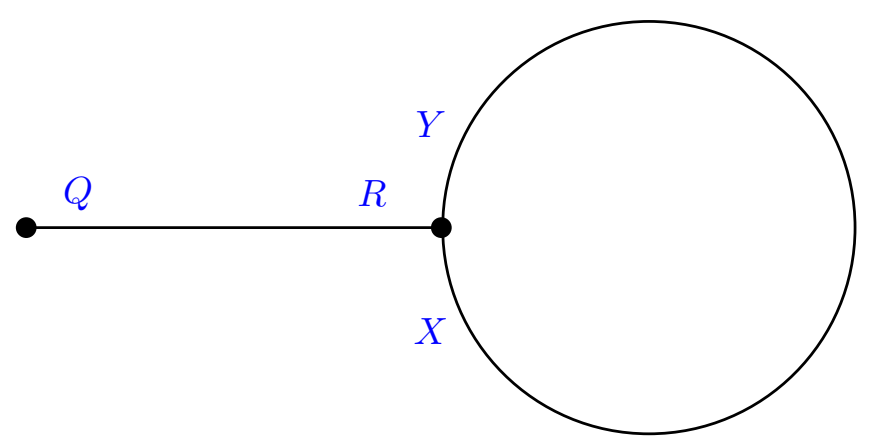

Figure 2: the group $\left\langle a, b, t \mid a^{Q}=b^{R}, t b^{X} t^{-1}=b^{Y}\right\rangle$

Theorem 1.2. Suppose that $G=\left\langle a, b, t \mid a^{Q}=b^{R}, t b^{X} t^{-1}=b^{Y}\right\rangle$ is 2-generated. Then $G$ maps onto $B S(Q X, Q Y)$ if and only if one (or more) of the gcd's $X \wedge Q Y, Y \wedge Q X$, $Q \wedge R$ equals 1 . 
We say that two groups are epi-equivalent if each is isomorphic to a quotient of the other (of course, any group epi-equivalent to a Hopfian group is isomorphic to it). Theorem 6.6 may be viewed as the determination of the GBS groups which are epi-equivalent to a Baumslag-Solitar group (see Corollary 6.7).

Recall [5] that $B S(m, n)$ is Hopfian if and only if $m$ or $n$ equals \pm 1 , or $m, n$ have the same set of prime divisors (Remark 6.10 contains a simple geometric argument showing non-Hopficity, illustrated on Figure 6). If $m, n$ are integers which are not prime and not coprime, and $B S(m, n)$ is not Hopfian, then (Corollary 6.8) there are infinitely many GBS groups which are epi-equivalent to $B S(m, n)$.

Theorem [6.6, together with a result from [12] or [14, also yields the following characterization of GBS groups $G$ which are large (some finite index subgroup maps onto a non-abelian free group): $G$ is large unless it is a quotient of some $B S(m, n)$ with $m, n$ coprime (see Corollary 6.5).

In Section 7 we use immersions of graphs of groups [1] (see also [7]) to find BaumslagSolitar subgroups in GBS groups. In particular, we show that, if $\frac{m}{n}$ is a non-zero rational number written in lowest terms with $m \neq \pm n$, and $G$ contains elements $a$ and $t$ satisfying $t a^{q m} t^{-1}=a^{q n}$ for some $q \geq 1$, then $G$ contains $B S(m, n)$. In other words, one may define the set of moduli of $G$ (see Section 2) in terms of its Baumslag-Solitar subgroups.

We deduce that a GBS group is residually finite if and only if it is solvable or virtually $F \times \mathbb{Z}$ with $F$ free (see [15] for Baumslag-Solitar groups). This is well-known (see [18] page 128), but we have not found a reference in the literature, so we provide a proof. Our argument shows that a GBS group is residually finite if and only if all its Baumslag-Solitar subgroups are.

We also determine under which conditions one may embed a Baumslag-Solitar group into another.

Theorem 1.3 (Theorem 7.8$)$. Assume that $(r, s) \neq( \pm 1, \pm 1)$. Then $B S(r, s)$ embeds into $B S(m, n)$ if and only if the following hold:

1. $\frac{r}{s}$ is a power of $\frac{m}{n}$;

2. if $m$ and $n$ are divisible by $p^{\alpha}$ but not by $p^{\alpha+1}$, with $p$ prime and $\alpha \geq 0$, then $r$ and $s$ are not divisible by $p^{\alpha+1}$; in particular, any prime dividing rs divides $m n$;

3. if $m$ or $n$ equals \pm 1 , so does $r$ or $s$.

The second condition says for instance that $B S(12,20)$ does not embed into $B S(6,10)$. It follows from the theorem that $B S(m, n)$ contains infinitely many non-isomorphic BaumslagSolitar groups if and only if $m \neq \pm n$. For instance, $B S(2,3)$ contains $B S\left(2^{a+b} 3^{c}, 2^{b} 3^{a+c}\right)$ for all $a, b, c \geq 0$, while $B S(3,3)$ only contains $\mathbb{Z}^{2}=B S(1,1)$.

At the end of the paper we characterize subgroups of $B S(n, n)$ and $B S(n,-n)$. In particular:

Theorem 1.4 (Corollary 8.2). A finitely generated group $G$ embeds into some $B S(n, n)$ if and only if $G$ is torsion-free, and either $G$ is free or $G$ is a central extension of $\mathbb{Z}$ by a free product of cyclic groups.

The paper is organized as follows.

Section 2 consists of preliminaries about GBS groups. We also use results from [13] in order to describe 2-generated GBS groups. Section 3 consists of generalities about maps between GBS groups. In particular, we show that (roughly speaking) a GBS group $G$ maps onto a Baumslag-Solitar group if and only if it is represented by labelled graphs with first Betti number at least 1 (see Lemma 3.6 for a correct statement), and we illustrate how strongly the descending chain condition fails for GBS groups. 
In Section 4 we prove Theorem 1.1; given a 2-generated GBS group $G$, we find the values of $m$ and $n$ for which there is an epimorphism $B S(m, n) \rightarrow G$. Corollaries are derived in Section 5 , in particular, we determine how many non-isomorphic GBS quotients a given $B S(m, n)$ has.

In Section 6 we state and prove Theorem 6.6, determining whether a given GBS group $G$ is epi-equivalent to a Baumslag-Solitar group (see Corollary 6.7). We also find which $B S(m, n)$ are epi-equivalent to infinitely many non-isomorphic GBS groups.

Section 7 is devoted to the construction of Baumslag-Solitar subgroups. In particular, we prove Theorem 1.3. In the last section we study subgroups of $B S(n, n)$.

This work was partially supported by ANR-07-BLAN-0141-01 and ANR-2010-BLAN116-03.

\section{Contents}

1 Introduction and statement of results 1

2 Preliminaries 4

2.1 Generalities on GBS groups . . . . . . . . . . . . . 4

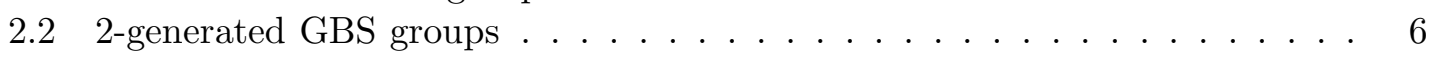

3 Maps between GBS groups 8

3.1 Elliptic-friendly homomorphisms . . . . . . . . . . . . . . . . 9

3.2 Epimorphisms between Baumslag-Solitar groups . . . . . . . . . . . . 9 9

3.3 Baumslag-Solitar quotients of GBS groups . . . . . . . . . . . . . . 10

3.4 Descending chains ........................ 11

4 Maps from Baumslag-Solitar groups 11

5 GBS quotients of $B S(m, n) \quad \mathbf{1 5}$

6 Groups epi-equivalent to a Baumslag-Solitar group 17

6.1 Contraction and displacement moves . . . . . . . . . . . . . 18

6.2 The case of a circle . . . . . . . . . . . . . . . . . . 19

6.3 The general case . . . . . . . . . . . . . . . 22

7 Baumslag-Solitar subgroups 2

7.1 Weakly admissible maps . . . . . . . . . . . . . . . . . . 23

7.2 Finding Baumslag-Solitar subgroups . . . . . . . . . . . . . . 24

7.3 Embeddings of Baumslag-Solitar groups . . . . . . . . . . . 26

8 Subgroups of $B S(n, n) \quad 29$

\section{Preliminaries}

\subsection{Generalities on GBS groups}

We refer to [9, 10, 12, for basic facts about GBS groups.

GBS groups are represented by labelled graphs. A labelled graph is a finite graph $\Gamma$ where each oriented edge $e$ has a label $\lambda_{e}$, a nonzero integer (possibly negative). When drawing pictures, we place $\lambda_{e}$ along $e$ near its origin (in blue).

We denote by $V$ the set of vertices of $\Gamma$, and by $\mathcal{E}$ the set of non-oriented edges. We view a non-oriented edge as $\varepsilon=(e, \tilde{e})$, where $\tilde{e}$ is $e$ with the opposite orientation. We 
denote by $v=o(e)$ the origin of $e$, and by $E_{v}$ the set of oriented edges with origin $v$. The cardinality $\left|E_{v}\right|$ of $E_{v}$ is the valence of $v$. A vertex is terminal if it has valence one.

We say that $\lambda_{e}$ is the label of e near the vertex $o(e)$, and that $\lambda_{e}$ is a label carried by $e$ or $\varepsilon$. There are $\left|E_{v}\right|$ labels near a vertex $v$.

We write $m \wedge n$ for the greatest common divisor (gcd) of two integers $m, n$ (i.e. the positive generator of $\langle m \mathbb{Z}, n \mathbb{Z}\rangle$ ); by convention, $m \wedge n>0$ regardless of the sign of $m, n$. On the other hand, $\operatorname{lcm}(m, n):=\frac{m n}{m \wedge n}$ may be negative.

A connected labelled graph defines a graph of groups. All edge and vertex groups are $\mathbb{Z}$, and the inclusion from the edge group $G_{e}$ to the vertex group $G_{o(e)}$ is multiplication by $\lambda_{e}$. The fundamental group $G$ of the graph of groups is the GBS group represented by $\Gamma$ (we do not always assume that $\Gamma$ is connected, but we implicitly do whenever we refer to the group it represents). The group $G$ may be presented as follows (see an example on Figure 2).

Choose a maximal subtree $\Gamma_{0} \subset \Gamma$. There is one generator $a_{v}$ for each vertex $v \in V$, and one generator $t_{\varepsilon}$ (stable letter) for each $\varepsilon$ in $\mathcal{E}_{0}$, the set of non-oriented edges not in $\Gamma_{0}$. Each non-oriented edge $\varepsilon=(e, \tilde{e})$ of $\Gamma$ contributes a relation $R_{\varepsilon}$. The relation is $\left(a_{o(e)}\right)^{\lambda_{e}}=\left(a_{o(\tilde{e})}\right)^{\lambda_{\tilde{e}}}$ if $\varepsilon$ is in $\Gamma_{0}$, and $t_{\varepsilon}\left(a_{o(e)}\right)^{\lambda_{e}} t_{\varepsilon}^{-1}=\left(a_{o(\tilde{e})}\right)^{\lambda_{\tilde{e}}}$ if $\varepsilon$ is not in $\Gamma_{0}$ (exchanging $e$ and $\tilde{e}$ amounts to replacing $t_{\varepsilon}$ by its inverse). This will be called a standard presentation of $G$, and the generating set will be called a standard generating set (associated to $\Gamma$ and $\left.\Gamma_{0}\right)$.

The group $G$ represented by $\Gamma$ does not change if one changes the sign of all labels near a given vertex $v$, or if one changes the sign of the labels $\lambda_{e}, \lambda_{\tilde{e}}$ carried by a given non-oriented edge $\varepsilon$. These will be called admissible sign changes.

A GBS group is elementary if it is isomorphic to $\mathbb{Z}$, or $\mathbb{Z}^{2}$, or the Klein bottle group $K=\left\langle x, y \mid x^{2}=y^{2}\right\rangle=\left\langle a, t \mid t a t^{-1}=a^{-1}\right\rangle$. These are the only virtually abelian GBS groups, and they have very special properties. Unless mentioned otherwise, our results apply to all GBS groups, but we do not always provide proofs for elementary groups.

A labelled graph $\Gamma$ is minimal if its Bass-Serre tree $T$ contains no proper $G$-invariant subtree; this is equivalent to no label near a terminal vertex being equal to \pm 1 . If $\Gamma$ is minimal, then $G$ is elementary if and only if $T$ is a point or a line.

The graph $\Gamma$ is reduced [8] if any edge $e$ such that $\lambda_{e}= \pm 1$ is a loop ( $e$ and $\tilde{e}$ have the same origin). Any labelled graph may be made reduced by a sequence of elementary collapses (see Figure 3); these collapses do not change $G$.
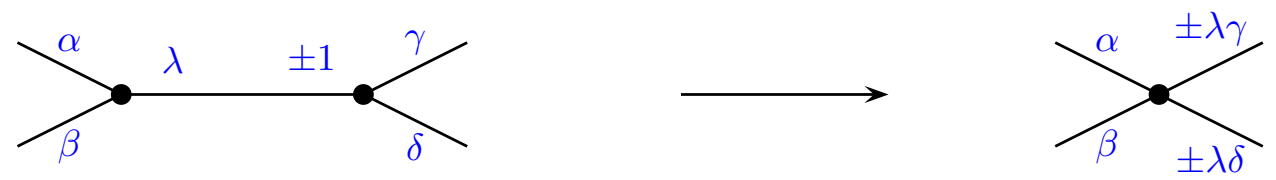

Figure 3: elementary collapse ( $G$ does not change)

There may be infinitely many reduced labelled graphs representing a given $G$. One case when uniqueness holds (up to admissible sign changes) is when $\Gamma$ is strongly slide-free [8]: if $e$ and $e^{\prime}$ are edges with the same origin, $\lambda_{e}$ does not divide $\lambda_{e^{\prime}}$.

In general, two reduced labelled graphs $\Gamma_{1}, \Gamma_{2}$ represent isomorphic GBS groups $G_{1}, G_{2}$ if and only if one can pass from $\Gamma_{1}$ to $\Gamma_{2}$ by a finite sequence of admissible sign changes, slide moves, induction moves, $\mathcal{A}^{ \pm 1}$-moves [4] (there is one exception to this fact, when 
$G_{1}=G_{2}=K$; the two presentations given above correspond to different reduced graphs: the first is an edge with labels 2 and 2 , the second a loop with labels 1 and -1 ).

We denote by $\beta(\Gamma)$ the first Betti number of $\Gamma$. If $G \neq K$, all labelled graphs representing $G$ have the same $\beta$, and we sometimes denote it by $\beta(G)$.

Let $G$ be represented by $\Gamma$. An element $g \in G$ is elliptic if it fixes a point in the Bass-Serre tree $T$ associated to $\Gamma$, or equivalently if some conjugate of $g$ belongs to a vertex group of $\Gamma$. All elliptic elements are pairwise commensurable (they have a common power). An element which is not elliptic is hyperbolic. If $G$ is non-elementary, ellipticity or hyperbolicity does not depend on the choice of $\Gamma$ representing $G$.

Any finitely generated subgroup of $G$ is free (if it acts freely on $T$ ) or is a GBS group (if the action is not free).

The quotient of $G$ by the subgroup generated by all elliptic elements may be identified with the topological fundamental group $\pi_{1}^{t o p}(\Gamma)$ of the graph $\Gamma$, a free group of $\operatorname{rank} \beta(\Gamma)$. In particular, any generating set of $G$ contains at least $\beta(\Gamma)$ hyperbolic elements.

If $G$ is non-elementary, there is a modular homomorphism $\Delta_{G}: G \rightarrow \mathbb{Q}^{*}$ associated to $G$. One may compute $\Delta_{G}(g)$ as follows: given any non-trivial elliptic element $a$, there is a non-trivial relation $g a^{m} g^{-1}=a^{n}$, and $\Delta_{G}(g)=\frac{m}{n}$ (given $g$, the numbers $m, n$ may depend on the choice of $a$, but $m / n$ does not). In particular, if $G=B S(m, n)=\langle a, t| t a^{m} t^{-1}=$ $\left.t^{n}\right\rangle$, then $\Delta_{G}$ sends $a$ to 1 and $t$ to $m / n$. Note that $h$ has to be elliptic is there is a relation $g h^{m} g^{-1}=h^{n}$ with $m \neq \pm n$ since its translation length in $T$ satisfies $|m| \ell(h)=|n| \ell(h)$.

Elliptic elements have modulus 1 , so $\Delta_{G}$ factors through $\pi_{1}^{t o p}(\Gamma)$ for any $\Gamma$ representing $G$. One may define $\Delta_{G}$ directly in terms of loops in $\Gamma$ : if $\gamma \in \pi_{1}^{t o p}(\Gamma)$ is represented by an edge-loop $\left(e_{1}, \ldots, e_{m}\right)$, then $\Delta_{G}(\gamma)=\prod_{i=1}^{m} \frac{\lambda_{e_{i}}}{\lambda_{\tilde{e}_{i}}}$. Note that $\Delta_{G}$ is trivial if $\Gamma$ is a tree.

$\Delta_{G}$ is trivial if and only if the center of $G$ is non-trivial. In this case the center is cyclic and only contains elliptic elements (see [12]); moreover, there is an epimorphism $G \rightarrow \mathbb{Z}$ whose kernel contains no non-trivial elliptic element (see Proposition 3.3 of [12]).

A non-elementary $G$ is unimodular if the image of $\Delta_{G}$ is contained in $\{1,-1\}$. This is equivalent to $G$ having a normal infinite cyclic subgroup, and also to $G$ being virtually $F_{n} \times \mathbb{Z}$ for some $n \geq 2$ (see [12]).

\section{$2.2 \quad 2$-generated GBS groups}

Any quotient of a Baumslag-Solitar group is 2-generated. Conversely, we note:

Lemma 2.1. Any 2-generated GBS group $G$ is a quotient of some Baumslag-Solitar group $B S(m, n)$.

Proof. We may assume that $G$ is non-elementary. There exists a generating pair $(a, t)$ with $a$ elliptic (this may be deduced from [13], or from the general fact that any finite generating set of a non-free group acting on a tree is Nielsen-equivalent to one containing an elliptic element). Since $a$ and $t a t^{-1}$ are commensurable, $a$ and $t$ satisfy a non-trivial relation $t a^{m} t^{-1}=a^{n}$ and $G$ is a quotient of $B S(m, n)$.

In Sections 4, 5, 6 we shall study GBS groups $G$ which are 2-generated, and not cyclic. Note that the isomorphism problem is solvable for these groups by 3 .

We always represent $G$ by a reduced labelled graph $\Gamma$. 2-generation implies that the sum of the first Betti number $\beta(\Gamma)$ and the number of terminal vertices $t(\Gamma)$ is at most 2. This is a consequence of Theorem 1.1 of [13, but it may be deduced directly from Grushko's theorem as follows. Add to a standard presentation of $G$ the relations $a_{v}=1$ if $v$ is not terminal, and $\left(a_{v}\right)^{p_{v}}=1$ if $v$ is terminal and $p_{v}$ is a prime dividing the label near $v$. This maps $G$ onto the free product of $\beta_{1}(\Gamma)+t(\Gamma)$ non-trivial cyclic groups, so $\beta_{1}(\Gamma)+t(\Gamma) \leq 2$.

We deduce that there are only three possibilities for the topological type of $\Gamma$ : it may be homeomorphic to a segment, a circle, or a lollipop. 
We picture the corresponding graphs on Figure 1, often viewing a circle as a special case of a lollipop. The number of edges is $k$ or $k+\ell$ respectively. Since $\Gamma$ is reduced, no label is equal to \pm 1 , except possibly $x_{0}$ and $y_{1}$ when $\ell=1$. We denote by $v_{i}$ the vertex next to the labels $q_{i}$ and/or $r_{i}$. In the lollipop case, the vertex next to the labels $x_{j}$ and/or $y_{j}$ is denoted by $w_{j}$; there is a special vertex $v_{k}=w_{0}=w_{\ell}$, we will usually denote it by $w_{0}$.

In the lollipop case, $G$ has a standard presentation with generators $a_{0}, \ldots, a_{i}, \ldots, a_{k-1}$ (associated to the vertices $v_{i}$ ) $, b_{0}, \ldots, b_{j}, \ldots, b_{\ell-1}$ (associated to the $w_{j}$ 's), $\tau$ (stable letter) and relations

$$
\begin{aligned}
& \left(a_{i}\right)^{q_{i}}=\left(a_{i+1}\right)^{r_{i+1}} \quad \text { for } i=0, \ldots, k-2 \\
& \left(a_{k-1}\right)^{q_{k-1}}=\left(b_{0}\right)^{r_{k}} \\
& \left(b_{j}\right)^{x_{j}}=\left(b_{j+1}\right)^{y_{j+1}} \quad \text { for } j=0, \ldots, \ell-2 \\
& \tau\left(b_{\ell-1}\right)^{x_{\ell-1}} \tau^{-1}=\left(b_{0}\right)^{y_{\ell}} .
\end{aligned}
$$

In the segment case, we delete $b_{1}, \ldots, b_{\ell-1}, \tau$ and relations (3) and (4), and we write $a_{k}$ instead of $b_{0}$. In the circle case, we delete $a_{0}, \ldots, a_{k-1}$ and relations (1) and (2).

Definition $2.2(\mathrm{Q}, \mathrm{R}, \mathrm{X}, \mathrm{Y})$. Given $\Gamma$ as above, we define numbers as follows:

- if $\Gamma$ is a segment, we let $Q=\prod_{i=0}^{k-1} q_{i}$ and $R=\prod_{i=1}^{k} r_{i}$;

- if $\Gamma$ is a lollipop, we let $Q=\prod_{i=0}^{k-1} q_{i}, R=\prod_{i=1}^{k} r_{i}, X=\prod_{j=0}^{\ell-1} x_{j}, Y=\prod_{j=1}^{\ell} y_{j}$ (with $Q=R=1$ if $\Gamma$ is a circle).

We now recall the definition of a plateau [13]. For $p$ a prime, a $p$-plateau is a nonempty connected subgraph $P \subset \Gamma$ such that, if $e$ is an oriented edge whose origin $v$ belongs to $P$, then the label $\lambda_{e}$ of $e$ near $v$ is divisible by $p$ if and only if $e$ is not contained in $P$. A plateau is a subgraph which is a $p$-plateau for some $p$. In particular, every terminal vertex is a plateau, and $\Gamma$ is a plateau. A plateau is interior if it contains no terminal vertex.

Theorem 1.1 of [13] states that the $\operatorname{rank} \operatorname{rk}(G)$ of $G$ (minimal cardinality of a generating set) is equal to $\beta(\Gamma)+\mu(\Gamma)$, with $\beta(\Gamma)$ the first Betti number and $\mu(\Gamma)$ the minimal cardinality of a set of vertices meeting every plateau. Thus:

Lemma 2.3. Let $\Gamma$ be a reduced labelled graph representing a 2-generated GBS group.

- If $\Gamma$ is not a circle, there is no interior plateau.

- If $\Gamma$ is a circle, there is a vertex belonging to every plateau.

Convention 2.4. When $\Gamma$ is a circle, we always choose the numbering of vertices so that $w_{0}$ belongs to every plateau.

With this convention, Lemma 2.3 implies:

Lemma 2.5. $\quad-q_{j}$ and $r_{i}$ are coprime for $1 \leq i \leq j \leq k-1$;

- If $\Gamma$ is a lollipop (possibly a circle), then $x_{j}$ and $y_{i}$ are coprime for $1 \leq i \leq j \leq \ell-1$; if a prime $p$ divides $R$, then $p$ divides $X$ or $Y$, but not both.

Proof. We argue by contradiction, showing the existence of a plateau contradicting Lemma 2.3. Suppose that a prime $p$ divides $q_{j}$ and $r_{i}$, with $i \leq j$ and $j-i$ minimal. Then the segment bounded by the vertices $v_{i}$ and $v_{j}$ is an interior $p$-plateau, a contradiction. In the lollipop case, the same argument applies to $x_{j}$ and $y_{i}$.

Now suppose that $p$ divides $r_{i}$, with $i$ maximal. If $p$ does not divide $X Y$, then $v_{i}$ is a boundary point of a $p$-plateau (containing the circle). If $p$ divides both $X$ and $Y$, say $p \mid x_{j}$ with $j \geq 0$ minimal and $p \mid y_{j^{\prime}}$ with $j^{\prime} \leq \ell$ maximal (and necessarily $j<j^{\prime}$ ), then $v_{i}$, $w_{j}$ and $w_{j^{\prime}}$ bound a $p$-plateau. 
Consider a standard presentation of a GBS group $G$, with generators $a_{v}$ and $t_{\varepsilon}$. Proposition 3.9 of [13] states that, given any subset $V_{1} \subset V$ meeting every plateau, $G$ is generated by the stable letters $t_{\varepsilon}$ and the $a_{v}$ 's for $v \in V_{1}$ (in other words, the $a_{v}$ 's for $v \notin V_{1}$ may be removed from the generating set).

Applied to a 2-generated $G$, this says that $G$ is generated by $a_{0}$ and $a_{k}$ in the segment case, by $a_{0}$ and $\tau$ in the lollipop case. If $\Gamma$ is a circle, the generators $a_{i}$ do not exist; $G$ is generated by $b_{0}$ and $\tau$ (assuming Convention 2.4).

Lemma 2.6. Let $\left(s, s^{\prime}\right)$ be a generating pair of $G$, with $s$ elliptic. Then $s$ is conjugate to $\left(a_{v}\right)^{r_{0}}$, with $r_{0} \in \mathbb{Z}$ and:

- if $\Gamma$ is a segment, $v$ is one of the terminal vertices $v_{0}$ or $v_{k}$ of $\Gamma$;

- if $\Gamma$ is a circle, $v$ belongs to every plateau;

- if $\Gamma$ is a lollipop, $v=v_{0}$ is the terminal vertex of $\Gamma$.

Moreover, $r_{0} \wedge p=1$ for each $p$ such that $v$ belongs to a proper $p$-plateau.

Remark 2.7. We may be more explicit about $r_{0}$, using the notations of Definition 2.2. If $\Gamma$ is a segment, then $r_{0} \wedge Q=1$ if $s$ is (up to conjugacy) a power of $a_{0}$, and $r_{0} \wedge R=1$ if $s$ is (up to conjugacy) a power of $a_{k}$. In the circle/lollipop case, $r_{0}$ is coprime with $Q X \wedge Q Y$ because $v$ belongs to a $p$-plateau $P$ with $w_{0} \notin P$ if and only if $p$ divides $Q$, to a proper $p$-plateau containing $w_{0}$ if and only if $p$ divides $X$ and $Y$ but not $Q$.

Proof. If $\Gamma$ is a segment of length $>1$, adding to a standard presentation the relations $a_{v}=1$ for $v$ not terminal yields a group of rank 2 (the free product of two nontrivial cyclic groups). It follows that $s$ must be conjugate to $\left(a_{v}\right)^{r_{0}}$ with $v$ terminal. If $v$ belongs to a proper $p$-plateau $P$, we add the relations $\left(a_{w}\right)^{p}=1$ for $w \in P$, and $a_{w}=1$ if $w \notin P$ and $w$ is not terminal. We obtain a group of rank 2 by Lemma 3.4 of [13], so $p$ does not divide $r_{0}$.

In the circle/lollipop case, $s$ is conjugate to $\left(a_{v}\right)^{r_{0}}$ with $v$ belonging to every plateau by Corollary 3.6 of [13] (which is proved also by adding relations killing powers of elliptic elements). Moreover, $r_{0}$ is not divisible by $p$ if $v$ belongs to a $p$-plateau by Corollary 3.7 of [13].

We conclude this subsection with the following useful fact.

Lemma 2.8. If a GBS group $G$ is 2-generated and is not a solvable $B S(1, n)$, it may be represented by a labelled graph $\Gamma$ with no label equal to \pm 1 .

Proof. Represent $G$ by a reduced $\Gamma$ with minimal number of edges. If some label equals \pm 1 , then $\Gamma$ is a lollipop with $\ell=1$ : the circle subgraph consists of a single edge with at least one label equal to \pm 1 , say $y_{1}= \pm 1$. Since $G$ is not solvable, $\Gamma$ is not a circle, so there is another edge $e$ attached to the vertex $w_{0}$, with label $r_{k}$. By Lemma 2.5, every prime dividing $r_{k}$ divides $x_{0}$. One may then perform induction moves (see [10, 3]) to make $r_{k}$ equal to \pm 1 . Collapsing the edge $e$ (as on Figure 3) yields a graph with one less edge representing $G$, a contradiction.

\section{Maps between GBS groups}

Lemma 3.1. Let $f: G \rightarrow G^{\prime}$ be a homomorphism between $G B S$ groups, with $f(G)$ nonelementary. If a is elliptic in $G$, then $f(a)$ is elliptic in $G^{\prime}$.

The result of [11] is a special case. 
Proof. (compare [8], proof of Corollary 6.10). Let $T$ be a tree on which $G^{\prime}$ acts with cyclic stabilizers. We let $G$ act on $T$ through $f$. By way of contradiction, suppose that $a$ acts hyperbolically on $T$. It has an axis $A$, the unique $a$-invariant line; it is also the axis of any power $a^{n}$ with $n \neq 0$. If $g \in G$, the elements $a$ and $g a g^{-1}$ have a common power, so $g$ leaves $A$ invariant. It follows that the line $A$ is $f(G)$-invariant, so $f(G)$ is elementary ( $\mathbb{Z}$, $\mathbb{Z}^{2}$, or $\left.K\right)$, a contradiction.

\subsection{Elliptic-friendly homomorphisms}

Lemma 3.2. Let $f: G \rightarrow G^{\prime}$ be a homomorphism from a non-elementary GBS group to a torsion-free group. Suppose that ker $f$ contains a non-trivial elliptic element. Then ker $f$ contains all elliptic elements. If $\Gamma$ is any labelled graph representing $G$, then $f$ factors through the topological fundamental group of $\Gamma$, and therefore $\beta(\Gamma) \geq \operatorname{rk}(f(G))$.

Proof. The first assertion holds because any two elliptic elements of $G$ are commensurable (they have a common power). If $\Gamma$ is any graph of groups, its topological fundamental group is the quotient of $G$ by the (normal) subgroup generated by all elliptic elements.

We say that $f$ is elliptic-friendly if ker $f$ contains no non-trivial elliptic element. Lemma 3.2 clearly implies:

Corollary 3.3. If $G$ is a non-elementary $G B S$ group with $\beta(G)=1$, and $G^{\prime}$ is torsionfree, any homomorphism $f: G \rightarrow G^{\prime}$ with non-cyclic image is elliptic-friendly.

Lemma 3.4. Let $f: G \rightarrow G^{\prime}$ be an elliptic-friendly homomorphism between GBS groups, with $G$ non-elementary.

If $f(G)$ is non-elementary, then $\Delta_{G}=\Delta_{G^{\prime}} \circ f$; in particular, $\Delta_{G}$ and $\Delta_{G^{\prime}}$ have the same image in $\mathbb{Q}^{*}$ if $f$ is onto.

If $f(G)$ is elementary, $\Delta_{G}$ is trivial when $f(G)$ is $\mathbb{Z}$ or $\mathbb{Z}^{2}$, has image contained in $\{1,-1\}$ when $f(G)$ is the Klein bottle group $K$.

See Section 2 for the definition of the modular homomorphism $\Delta_{G}: G \rightarrow \mathbb{Q}^{*}$.

Proof. If $\Delta_{G}(g)=\frac{m}{n}$, there is a non-trivial elliptic element $a \in G$ such that $g a^{m} g^{-1}=a^{n}$. One deduces $f(g) f(a)^{m} f(g)^{-1}=f(a)^{n}$, with $f(a)$ non-trivial.

This equation implies $\Delta_{G^{\prime}}(f(g))=\frac{m}{n}$ if $f(G)$ is non-elementary, because $f(a)$ is elliptic by Lemma 3.1. It implies $m=n$ in $\mathbb{Z}$ and $\mathbb{Z}^{2}, m= \pm n$ in $K$.

\subsection{Epimorphisms between Baumslag-Solitar groups}

Lemma 3.5. There is an epimorphism $f: B S(m, n) \rightarrow B S\left(m^{\prime}, n^{\prime}\right)$ if and only if one of the following holds:

- $(m, n)$ is an integral multiple of $\left(m^{\prime}, n^{\prime}\right)$ or $\left(n^{\prime}, m^{\prime}\right)$;

- $B S\left(m^{\prime}, n^{\prime}\right)=B S(1,-1)$ is the Klein bottle group $K$, and $m=n$ with $m$ even.

This is Proposition A.11 of [16] or Theorem 5.2 of [6] in the non-elementary case.

Proof. Write $G=B S(m, n)=\left\langle a, t \mid t a^{m} t^{-1}=a^{n}\right\rangle$ and $G^{\prime}=B S\left(m^{\prime}, n^{\prime}\right)=\left\langle a^{\prime}, t^{\prime}\right|$ $\left.t^{\prime} a^{\prime m^{\prime}} t^{\prime-1}=a^{\prime n^{\prime}}\right\rangle$.

The "if" direction is clear, noting the epimorphism from $B S(2 m, 2 m)$ to $B S(1,-1)$ mapping $t$ to $a^{\prime}$ and $a$ to $t^{\prime}$.

For the converse, we first suppose that $B S(m, n)$ and $B S\left(m^{\prime}, n^{\prime}\right)$ are non-elementary (i.e. different from $\mathbb{Z}^{2}$ and $K$ ). If $f$ exists, it is elliptic-friendly by Corollary [3.3, so $\frac{m}{n}$ equals $\frac{m^{\prime}}{n^{\prime}}$ or $\frac{n^{\prime}}{m^{\prime}}$ by Lemma 3.4. If $m \neq n$, abelianizing shows that $m-n$ is divisible by 
$m^{\prime}-n^{\prime}$. If $m=n$, dividing by the center $\left\langle a^{m}\right\rangle$ shows that $m$ is divisible by $m^{\prime}$. The result follows.

The case when $G$ is elementary is easy and left to the reader (any epimorphism from $G$ to a non-cyclic GBS group is an isomorphism), so assume that $G^{\prime}$ is elementary and $G$ is not. If $G^{\prime}=\mathbb{Z}^{2}$, Lemma 3.4 implies $m=n$ as required. If $G^{\prime}=K$, it implies $m= \pm n$ and we have to rule out the possibility that $m=n$ with $m$ odd.

Assume $m=n$ and consider $\chi: G^{\prime} \rightarrow \mathbb{Z} / 2 \mathbb{Z} \times \mathbb{Z} / 2 \mathbb{Z}$ obtained by abelianizing and killing the image of the center $\left\langle t^{\prime 2}\right\rangle$. Since $a^{m}$ is central in $B S(m, m)$, its image by $f$ is killed by $\chi$. If $m$ is odd, the element $a$ itself is killed by $\chi \circ f$. This is a contradiction since $\chi \circ f$ is onto, but $G /\langle\langle a\rangle\rangle \simeq \mathbb{Z}$ does not map onto $\mathbb{Z} / 2 \mathbb{Z} \times \mathbb{Z} / 2 \mathbb{Z}$.

\subsection{Baumslag-Solitar quotients of GBS groups}

Lemma 3.6. Let $G$ be a non-elementary GBS group, and write $\beta=\beta(G)$.

- If $\beta=0$, then $G$ has no quotient isomorphic to a Baumslag-Solitar group, except possibly to $K=B S(1,-1)$.

- If $\beta \geq 1$, then $G$ has a quotient isomorphic to a Baumslag-Solitar group. There exists an elliptic-friendly epimorphism $f$ from $G$ to a Baumslag-Solitar group if and only if the image of $\Delta_{G}$ is cyclic (possibly trivial).

Proof. Let $\Gamma$ be any labelled graph representing $G$.

If $\beta=0$, then $G$ may be generated by a set of elements which are all commensurable with each other, and any quotient of $G$ inherits this property. This rules out all BaumslagSolitar groups except $K=\left\langle u, v \mid u^{2}=v^{2}\right\rangle$.

Now suppose $\beta=1$. The image of $\Delta_{G}$ is cyclic, and we construct an elliptic-friendly epimorphism as follows. The graph $\Gamma$ contains a unique embedded circle. Choose an edge on this circle, and collapse all other edges to a point. This expresses $G$ as an HNN extension where the base group $G_{0}$ is a GBS group represented by a labelled graph which is a tree, and the amalgamated subgroups are cyclic and consist of elliptic elements of $G_{0}$. There is an elliptic-friendly epimorphism from $G_{0}$ to $\mathbb{Z}$ (see Proposition 3.3 of [12]). It extends to an elliptic-friendly epimorphism from $G$ to some $B S(m, n)$.

If $\beta \geq 2$, then $G$ maps onto $F_{2}$ hence onto any Baumslag-Solitar group. If there is an elliptic-friendly epimorphism $f: G \rightarrow B S(m, n)$, the image of $\Delta_{G}$ is cyclic by Lemma 3.4 .

Conversely, we suppose that the image of $\Delta_{G}$ is cyclic, contained in the subgroup of $\mathbb{Q}^{*}$ generated by $\frac{m}{n}$ (with $m$ and $n$ coprime), and we now construct an elliptic-friendly epimorphism $f: G \rightarrow B S(m, n)$ as in the case $\beta=1$. We choose a maximal subtree $\Gamma_{0} \subset \Gamma$ and we map the GBS group $G_{0}$ represented by $\Gamma_{0}$ to $\mathbb{Z}$ without killing the elliptic elements. This yields a quotient $G^{\prime}$ of $G$ with presentation $\left\langle a, t_{1}, \ldots, t_{\beta} \mid t_{i} a^{m_{i}} t_{i}^{-1}=a^{n_{i}}\right\rangle$. Each $\frac{m_{i}}{n_{i}}$ is in the image of $\Delta_{G}$, so we may write $\left(m_{i}, n_{i}\right)=\left(\theta_{i} m^{\kappa_{i}}, \theta_{i} n^{\kappa_{i}}\right)$ or $\left(m_{i}, n_{i}\right)=\left(\theta_{i} n^{\kappa_{i}}, \theta_{i} m^{\kappa_{i}}\right)$ with integers $\theta_{i} \neq 0$ and $\kappa_{i} \geq 0$. We now map $G^{\prime}$ to $B S(m, n)=\left\langle a, t \mid t a^{m} t^{-1}=a^{n}\right\rangle$ by sending $a$ to $a$ and $t_{i}$ to $t^{ \pm \kappa_{i}}$. This is onto because the image of $\Delta_{G}$ in $\mathbb{Q}^{*}$ is generated by the numbers $\frac{m_{i}}{n_{i}}$.

Example 3.7. Some GBS groups with $\beta=0$ map onto $K$, some do not. For instance, let us show that $G=\left\langle a, b \mid a^{m}=b^{n}\right\rangle$ maps onto $K$ if and only if $m$ and $n$ are even and divisible by the same powers of 2 (i.e. $m=2 \lambda r$ and $n=2 \lambda s$ with $r, s$ odd and coprime).

We view $K$ as $\left\langle u, v \mid u^{2}=v^{2}\right\rangle$. If $m=2 \lambda r$ and $n=2 \lambda s$ as above, we map $G$ to $K$ by sending $a$ to $u^{s}$ and $b$ to $v^{r}$ (to see that $u^{s}$ and $v^{r}$ generate $K$, write $x r+y s=1$ and observe that $x$ or $y$ must be even).

Conversely, suppose $f$ is an epimorphism from $G$ to $K$. Since $a^{m}$ is central, $f\left(a^{m}\right)$ is a power of $u^{2}$, so $f(a)$ is conjugate to a power of $u$ or $v$, and similarly for $f(b)$. Abelianizing shows that $f(a)$ is conjugate to an odd power of $u$, and $f(b)$ to an odd power of $v$ (up to 
swapping $u$ and $v$ ). In particular, $m$ and $n$ are even. The relation $a^{m}=b^{n}$ forces $m$ and $n$ to be divisible by the same powers of 2 .

Example 3.8. It is not true that every GBS group with $\beta=1$ maps onto a BaumslagSolitar group different from $K$. For instance, $K$ is the only Baumslag-Solitar quotient of $G=\left\langle a, b, t \mid a^{2}=b^{2}, t b t^{-1}=b^{-1}\right\rangle$ : if $G$ maps onto $B S(m, n)$, Lemma 3.4 implies $m=-n$; abelianizing shows $m= \pm 1$.

\subsection{Descending chains}

Since Baumslag-Solitar groups may fail to be Hopfian, they do not satisfy the descending chain condition: there exists an infinite sequence of non-injective epimorphisms $f_{n}: G_{n} \rightarrow$ $G_{n+1}$. On the other hand, Baumslag-Solitar groups satisfy the descending chain condition if we require $G_{n}$ and $G_{n+1}$ to be non-isomorphic (see Lemma 3.5).

Among GBS groups, the descending chain condition fails in the strongest possible way.

Proposition 3.9. There exists a sequence $\left(f_{n}\right)_{n \geq 1}$ of epimorphisms $f_{n}: G_{n} \rightarrow G_{n+1}$ such that:

- each $G_{n}$ is a GBS group which is a quotient of $B S(18,36)$ and maps onto $B S(9,18)$;

- there is no epimorphism $G_{n+1} \rightarrow G_{n}$.

In particular, $G_{n}$ and $G_{n+1}$ are not isomorphic, and not even epi-equivalent.

Proof. Let $G_{n}=\left\langle a, b, t \mid a^{6}=b^{2^{n}}, t b^{3} t^{-1}=b^{6}\right\rangle$ (it is represented by a lollipop as on Figure 2 with $Q=6$ ). It is generated by $a$ and $t$, which satisfy $t a^{18} t^{-1}=a^{36}$, so is a quotient of $B S(18,36)$. Constructing epimorphisms from $G_{n}$ to $G_{n+1}$, and from $G_{n}$ to $B S(9,18)$, is easy and left to the reader. We assume that there is an epimorphism $f: G_{n+1} \rightarrow G_{n}$, and we argue towards a contradiction.

We denote by $a^{\prime}, b^{\prime}, t^{\prime}$ the generators of $G_{n+1}$. Since $\left(f\left(a^{\prime}\right), f\left(t^{\prime}\right)\right)$ is a generating pair of $G_{n}$ with $f\left(a^{\prime}\right)$ elliptic, Lemma 2.6 and Remark 2.7 imply that $f\left(a^{\prime}\right)$ is (conjugate to) $a^{r}$ with $r$ coprime with 6 . Since $\left(a^{\prime}\right)^{6}=\left(b^{\prime}\right)^{2^{n+1}}$, the element $c=f\left(\left(a^{\prime}\right)^{6}\right)=a^{6 r}=b^{2^{n} r}$ has a $2^{n+1}$-th root $u$ in $G_{n}$. The group $A=\langle a, b\rangle$ is a vertex group in a (non-GBS) splitting of $G_{n}$, with incident edge groups conjugate to $\left\langle b^{3}\right\rangle$ and $\left\langle b^{6}\right\rangle$. Since $c=b^{2^{n} r}$ is central in $A$, and $r$ is not divisible by $3, c$ fixes a unique point in the Bass-Serre tree of this splitting, so $u \in A$. Now consider the epimorphism from $A$ to $\mathbb{Z}$ mapping $a$ to $2^{n-1}$ and $b$ to 3 . The image of $c$ is $3 r 2^{n}$, so $c$ does not have a $2^{n+1}$-th root in $A$ since $r$ is odd. We have reached the desired contradiction.

\section{Maps from Baumslag-Solitar groups}

We have seen (Lemma 2.1) that any 2-generated GBS group $G$ is a quotient of some $B S(m, n)$. In this section we fix $G$ and we determine for which values of $m$ and $n$ this happens.

Theorem 4.1. Let $G$ be a 2-generated GBS group, represented by a reduced labelled graph $\Gamma$ as on Figure 1. Assume that $G$ is not cyclic or the Klein bottle group $K$.

- If $\Gamma$ is a segment, then $G$ is a quotient of $B S(m, n)$ if and only if $m=n$, and $m$ is divisible by $Q$ or $R$.

- If $\Gamma$ is a lollipop (possibly a circle), then $G$ is a quotient of $B S(m, n)$ if and only if $(m, n)$ is an integral multiple of $(Q X, Q Y)$ or $(Q Y, Q X)$. 
In the lollipop case, there is a unique "smallest" Baumslag-Solitar group mapping onto $G$, namely $B S(Q X, Q Y)$. In the segment case, there are in general two groups, $B S(Q, Q)$ and $B S(R, R)$.

We establish a lemma before starting the proof.

Lemma 4.2. Let $\Gamma$ be a labelled graph homeomorphic to a segment, as on Figure 1, with associated group $G=\left\langle a_{0}, \ldots, a_{k} \mid\left(a_{i}\right)^{q_{i}}=\left(a_{i+1}\right)^{r_{i+1}}, i=0, \ldots, k-1\right\rangle$. Given $r_{0} \neq 0$, let $\left(a_{0}\right)^{r_{0} N}$ be a generator of $\left\langle\left(a_{0}\right)^{r_{0}}\right\rangle \cap\left\langle a_{k}\right\rangle$. Then:

1. $\left(a_{0}\right)^{q_{0} \cdots q_{j-1}}=\left(a_{j}\right)^{r_{1} \cdots r_{j}}$ for $j=1, \ldots, k$. In particular, $N$ divides $q_{0} \cdots q_{k-1}$.

2. If $q_{j}$ and $r_{i}$ are coprime whenever $0 \leq i \leq j \leq k-1$, then $N= \pm q_{0} \cdots q_{k-1}$.

3. If $p$ is a prime which divides no $q_{j} \wedge r_{i}$ with $0 \leq i \leq j \leq k-1$, then $N=q_{0} \cdots q_{k-1} / \theta$ with $\theta$ not divisible by $p$.

4. Let $p$ be prime, $\alpha \geq 0$, and $r_{0}=1$. Suppose that each $q_{j}$ for $0 \leq j \leq k-1$ and each $r_{i}$ for $1 \leq i \leq k$ is divisible by $p^{\alpha}$ but not by $p^{\alpha+1}$. Then $N$ is divisible by $p^{\alpha}$ but not by $p^{\alpha+1}$.

The fourth assertion will be used only in Subsection 7.3 .

Remark. $\left\langle a_{0}\right\rangle \cap\left\langle a_{k}\right\rangle$ is the center of $G$.

Proof. For the first assertion, we simply use the relations $\left(a_{i}\right)^{q_{i}}=\left(a_{i+1}\right)^{r_{i+1}}$. We show 3 (which clearly implies 2) by induction on $k$. Let $\theta_{j} \in \mathbb{Z}$ be such that $\left\langle\left(a_{0}\right)^{r_{0}}\right\rangle \cap\left\langle a_{j}\right\rangle$ is generated by $\left(a_{0}\right)^{r_{0} q_{0} \cdots q_{j-1} / \theta_{j}}=\left(a_{j}\right)^{r_{0} r_{1} \cdots r_{j} / \theta_{j}}$, with $\theta_{0}=1$. We must show that $\theta_{k}$ is not divisible by $p$. write

Using $\left\langle\left(a_{0}\right)^{r_{0}}\right\rangle \cap\left\langle a_{k}\right\rangle=\left\langle\left(a_{0}\right)^{r_{0}}\right\rangle \cap\left\langle\left(a_{k-1}\right)^{q_{k-1}}\right\rangle$, and the formula $m \mathbb{Z} \cap n \mathbb{Z}=\frac{m n}{m \wedge n} \mathbb{Z}$, we

$$
\begin{gathered}
\left\langle\left(a_{0}\right)^{r_{0}}\right\rangle \cap\left\langle a_{k}\right\rangle=\left\langle\left(a_{0}\right)^{r_{0}}\right\rangle \cap\left\langle a_{k-1}\right\rangle \cap\left\langle\left(a_{k-1}\right)^{q_{k-1}}\right\rangle=\left\langle\left(a_{k-1}\right)^{r_{0} \cdots r_{k-1} / \theta_{k-1}}\right\rangle \cap\left\langle\left(a_{k-1}\right)^{q_{k-1}}\right\rangle \\
=\left\langle\left(a_{k-1}\right)^{\frac{r_{0} \cdots r_{k-1} q_{k-1}}{\theta_{k-1} \theta^{\prime}}}\right\rangle=\left\langle\left(a_{k}\right)^{r_{0} \cdots r_{k-1} r_{k} / \theta_{k-1} \theta^{\prime}}\right\rangle
\end{gathered}
$$

with $\theta^{\prime}$ the gcd of $r_{0} \cdots r_{k-1} / \theta_{k-1}$ and $q_{k-1}$.

If $p$ is as in Assertion 3, then $\theta_{k-1}$ is not divisible by $p$ by induction, and neither is $\theta^{\prime}$, so $\theta_{k}= \pm \theta_{k-1} \theta^{\prime}$ is not divisible by $p$.

Assertion 4 is equivalent to saying that the largest power of $p$ dividing $\theta_{k}$ is $p^{(k-1) \alpha}$. We prove this by induction on $k$, noting that $\theta_{1}=1$. By induction the largest power of $p$ dividing $r_{0} \cdots r_{k-1} / \theta_{k-1}$ is $p^{\alpha}$, so the same is true for $\theta^{\prime}$ and the result follows since $\theta_{k}= \pm \theta_{k-1} \theta^{\prime}$.

The same proof shows:

Lemma 4.3. Let $v_{0}, \ldots, v_{k}$ be a segment of length $k$ in the Bass-Serre tree $T$ associated to some labelled graph. Let $a_{i}$ be a generator of the stabilizer of $v_{i}$, let $c_{i}$ be a generator of the stabilizer of the edge $v_{i} v_{i+1}$, and define $q_{0}, \ldots, q_{k-1}, r_{1}, \ldots r_{k}$, by $c_{i}=\left(a_{i}\right)^{q_{i}}=\left(a_{i+1}\right)^{r_{i+1}}$. Given $r_{0} \neq 0$, the conclusions of Lemma 4.3 hold for the index $N$ of $\left\langle\left(a_{0}\right)^{r_{0}}\right\rangle \cap\left\langle a_{k}\right\rangle$ in $\left\langle\left(a_{0}\right)^{r_{0}}\right\rangle$.

We can now prove the main result of this section.

Proof of Theorem 4.1. We assume that $G$ is not a Baumslag-Solitar group (the theorem follows from Lemma 3.5 if it is). In particular, $G$ is non-elementary, and so is any $B S(m, n)$ which maps onto $G$. We fix a presentation $\left\langle a, t \mid t a^{m} t^{-1}=a^{n}\right\rangle$ for $B S(m, n)$. 
- First suppose that $\Gamma$ is a segment. Since $G$ is 2-generated, it is generated by $a_{0}$ and $a_{k}$. These elements satisfy $\left(a_{0}\right)^{Q}=\left(a_{k}\right)^{R}$ by Lemma 4.2, so $G$ is a quotient of $B S(\alpha Q, \alpha Q)$ and $B S(\alpha R, \alpha R)$ for any integer $\alpha$ : send $a$ to $a_{0}$ and $t$ to $a_{k}$, or $a$ to $a_{k}$ and $t$ to $a_{0}$.

Conversely, suppose that $f$ is an epimorphism from $B S(m, n)$ to $G$. It is ellipticfriendly by Corollary 3.3 , so $m=n$ by Lemma 3.4 since $G$ has trivial modulus. The element $f(a)$ is elliptic, so by Lemma 2.6 we may assume (by composing $f$ with an inner automorphism of $G$ ) that $f(a)$ is a power of $a_{0}$ or $a_{k}$, say $f(a)=\left(a_{0}\right)^{r_{0}}$ with $r_{0} \neq 0$. In this case we show that $m$ is a multiple of $Q$ (it is a multiple of $R$ if $f(a)$ is a power of $a_{k}$ ).

We will apply Assertion 2 of Lemma 4.2, so we now check that $q_{j}$ and $r_{i}$ are coprime for $0 \leq i \leq j \leq k-1$ : this follows from Lemma 2.5 for $i>0$ (note that $r_{0}$ is not a label of $\Gamma$ ), and from Remark 2.7 for $i=0$.

The element $a^{m}$ is central in $B S(m, m)$, so $\left(a_{0}\right)^{r_{0} m}$ is central in $G$. In particular, it must be a power of $a_{k}$ (since $\left|r_{k}\right|>1$, the centralizer of $a_{k}$ is $\left\langle a_{k}\right\rangle$ ). Thus $\left(a_{0}\right)^{r_{0} m}$ belongs to $\left\langle\left(a_{0}\right)^{r_{0}}\right\rangle \cap\left\langle a_{k}\right\rangle$, which is generated by $\left(a_{0}\right)^{r_{0} Q}$ by Lemma 4.2, so $m$ is a multiple of $Q$.

- We now suppose that $\Gamma$ is a lollipop. We use the standard presentation $G=$ $\left\langle a_{0}, \ldots, a_{k-1}, b_{0}, b_{1}, \ldots, b_{\ell-1}, \tau\right\rangle$ described in Subsection 2.2. Since $G$ is 2-generated, it is generated by $a_{0}$ and $\tau$ (by $b_{0}$ and $\tau$ if $\Gamma$ is a circle). To unify notation, we will write $g_{0}$ for $a_{0}$ if $\Gamma$ is not a circle, for $b_{0}$ if $\Gamma$ is a circle, and we denote by $z_{0}$ the associated vertex $v_{0}$ or $w_{0}$.

The first assertion of Lemma 4.3 yields $\left(a_{0}\right)^{Q}=\left(b_{0}\right)^{R}$ and $\tau\left(b_{0}\right)^{X} \tau^{-1}=\left(b_{0}\right)^{Y}$, so that $\tau\left(g_{0}\right)^{Q X} \tau^{-1}=\left(g_{0}\right)^{Q Y}$. It follows that $G$ is a quotient of $B S(Q X, Q Y)$.

Conversely, consider an epimorphism $f: B S(m, n) \rightarrow G$. It is elliptic-friendly by Corollary 3.3, and $\frac{m}{n}$ equals $\frac{X}{Y}$ or $\frac{Y}{X}$ by Lemma 3.4. Also note that $(f(a), f(t))$ is a generating pair of $G$, with $f(a)$ elliptic.

By Lemma 2.6, the element $f(a)$ belongs, up to conjugacy, to the group carried by a vertex belonging to every plateau, so we may assume (by composing with an inner automorphism, and renumbering vertices if $\Gamma$ is a circle) that $f(a)=\left(g_{0}\right)^{r_{0}}$ with $r_{0} \neq 0$ (as above, beware that $r_{0}$ is not a label of $\Gamma$ ). By Remark 2.7 $r_{0}$ and $Q X \wedge Q Y$ are coprime.

Killing all elliptic elements defines an infinite cyclic quotient $Z$ of $G$, which is the topological fundamental group of $\Gamma$. The elements $a_{i}, b_{j}, f(a)$ vanish in $Z$, while $\tau$ and $f(t)$ map to generators. We may assume that $\tau$ and $f(t)$ map to the same generator. This implies $\frac{m}{n}=\frac{X}{Y}$. We have to show that $(m, n)$ is an integral multiple of $(Q X, Q Y)$.

We consider the Bass-Serre tree $T$ of $\Gamma$. We also consider the topological universal covering $\tilde{\Gamma}$, a line with segments of length $k$ attached (see Figure 4). The group $G$ acts on $\tilde{\Gamma}$ through $Z$. There is a natural equivariant map $\varphi: T \rightarrow \tilde{\Gamma}$ sending vertex to vertex and edge to edge; composing $\varphi$ with the covering map $\tilde{\Gamma} \rightarrow \Gamma$ yields the quotient map $\pi: T \rightarrow \Gamma=T / G$.

Since $G$ is not a solvable Baumslag-Solitar group, no label near the vertex $z_{0}$ of $\Gamma$ carrying $g_{0}$ equals \pm 1 , so $g_{0}$ fixes a unique point $u$ in $T$. This point is fixed by $f(a)=$ $\left(g_{0}\right)^{r_{0}}$. We have $t^{-1} a^{n}=a^{m} t^{-1}$, so $f\left(a^{m}\right)=\left(g_{0}\right)^{m r_{0}}$ fixes a point $u^{\prime} \in T$ such that $\varphi\left(u^{\prime}\right)=f\left(t^{-1}\right) \varphi(u)$, for instance $f\left(t^{-1}\right) u$. Among all such points $u^{\prime}$, we choose one at minimal distance from $u$; note that $u^{\prime}$ necessarily belongs to the $G$-orbit of $u$. We claim that $\varphi$ is then injective on the segment $u u^{\prime}$ (i.e. the map from $u u^{\prime}$ to $\Gamma$ is locally injective).

If not, there is a point $u^{\prime \prime} \in T$ between $u$ and $u^{\prime}$ such that the initial edges $e$ and $e^{\prime}$ of $u^{\prime \prime} u$ and $u^{\prime \prime} u^{\prime}$ have the same projection in $\Gamma$, so some $g \in G$ in the stabilizer of $u^{\prime \prime}$ maps $e^{\prime}$ to $e$. Now consider $g u^{\prime}$. It is closer to $u$ than $u^{\prime}$, and it has the same image as $u^{\prime}$ in $\tilde{\Gamma}$ since $g$ acts as the identity on $\tilde{\Gamma}$ (it is elliptic, so vanishes in $Z$ ). The element $\left(g_{0}\right)^{m r_{0}}$ fixes $u$ and $u^{\prime}$, hence $u^{\prime \prime}$, so it commutes with $g$ and therefore fixes $g u^{\prime}$. This contradicts the choice of $u^{\prime}$ and proves the injectivity claim.

Since $\varphi\left(u^{\prime}\right)=f\left(t^{-1}\right) \varphi(u)=\tau^{-1} \varphi(u)$, the projection of $u u^{\prime}$ to $\Gamma$ is a loop going once 


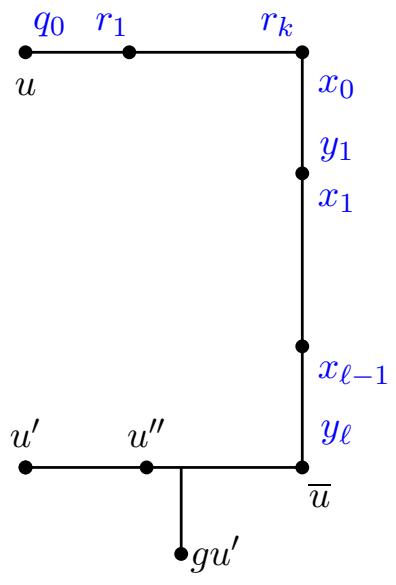

the segment $u u^{\prime} \subset T$
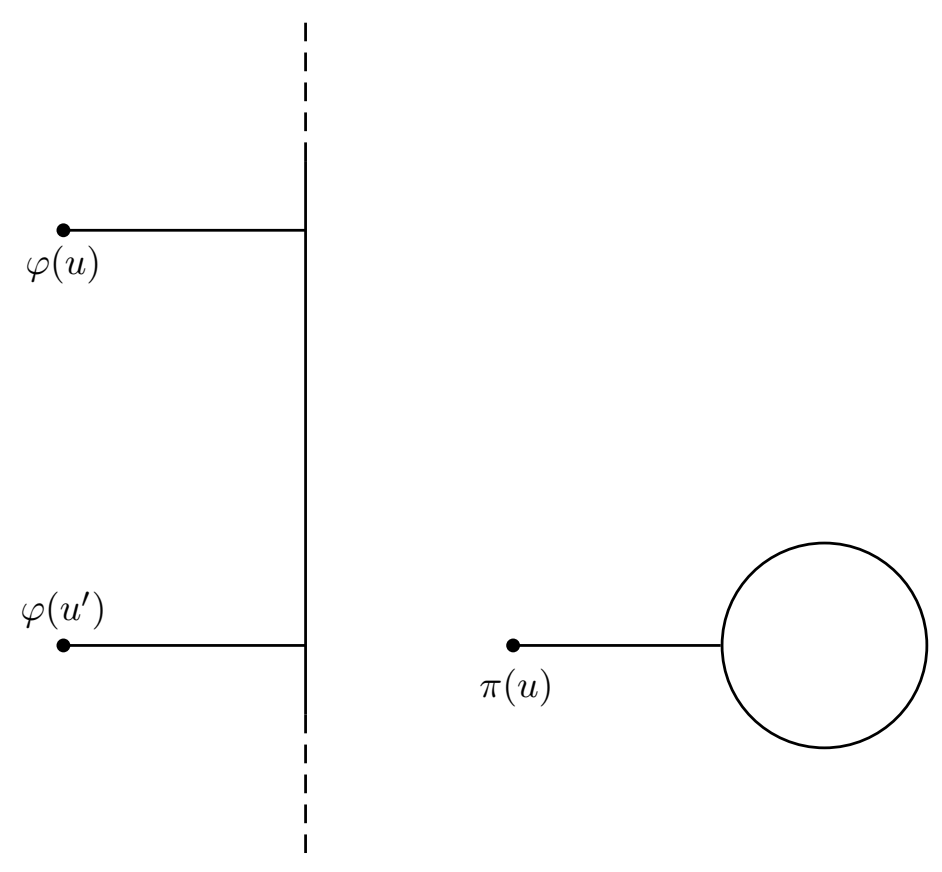

Figure 4

around the circle, so it is the immersed path of length $\ell+2 k$

$$
v_{0}, \ldots, v_{k-1}, v_{k}=w_{0}, \ldots, w_{\ell-1}, w_{\ell}=w_{0}=v_{k}, v_{k-1}, \ldots, v_{0}
$$

We wish to apply Lemma 4.3 to the segment $u u^{\prime}$, knowing that $g_{0}$ generates the stabilizer of $u$ and $\left(g_{0}\right)^{m r_{0}}$ fixes the whole segment. Recall that we want $(m, n)$ to be a multiple of $(Q X, Q Y)$.

First suppose that $\Gamma$ is a circle (so $u u^{\prime}$ has length $\ell$, and $g_{0}=b_{0}$ ). We know that $x_{j}$ and $y_{i}$ are coprime for $1 \leq i \leq j \leq \ell-1$ by Lemma 2.5, but $r_{0}$ does not have to be coprime with $X$, so we cannot apply the second assertion of Lemma 4.3. we will need the third one.

Our goal is to show that $\frac{m}{X}=\frac{n}{Y}$ is an integer (since $Q=1$ ). If it is not, write it in lowest terms and consider a prime $p$ dividing the denominator. It divides $X$ and $Y$, so it does not divide $r_{0}$, as explained above. Assertion 3 of Lemma 4.3 then implies that $m$ is a multiple of $X / \theta$ with $\theta$ not divisible by $p$, a contradiction.

In the lollipop case, we consider $p$ dividing the denominator of $\frac{m}{Q X}=\frac{n}{Q Y}$. We will apply Lemma 4.3 to the initial segment $u \bar{u}$ of length $k+\ell$ of $u u^{\prime}$. We know that $q_{j}$ and $r_{i}$ are coprime for $0 \leq i \leq j \leq k-1$ (recall that $r_{0}$ and $Q$ are coprime), and $x_{j}, y_{i}$ are coprime for $1 \leq i \leq j \leq \ell-1$, but we do not know that $r_{i}$ and $x_{j}$ are coprime.

If $p$ does not divide $r_{i} \wedge x_{j}$ for $0 \leq i \leq k$ and $0 \leq j \leq \ell-1$, Assertion 3 of Lemma 4.3 applies and gives a contradiction as above: $m$ is a multiple of $Q X / \theta$ with $\theta$ not divisible by $p$.

The condition that $p$ does not divide $r_{i} \wedge x_{j}$ certainly holds if $p$ does not divide $X$. By symmetry of the statement of Theorem 4.1, we may therefore assume that $p$ divides both $X$ and $Y$. It follows that $p$ does not divide $r_{0}$, and also that it does not divide $R=\prod_{i=1}^{k} r_{i}$ by the second assertion of Lemma 2.5. Thus the condition also holds in this case. 


\section{GBS quotients of $B S(m, n)$}

In the previous section we fixed $G$ and we determined for which values of $m, n$ one may view $G$ as a quotient of $B S(m, n)$. We now fix $m, n$ and we study the set of all GBS groups $G$ which are quotients of $B S(m, n)$, using Theorem 4.1. We assume $G \neq B S(1, \pm 1)$ (the results are trivial in these two cases).

We always represent $G$ by a reduced labelled graph $\Gamma$.

Proposition 5.1. Let $\Gamma$ be a reduced labelled graph representing a GBS group $G$ which is a quotient of $B S(m, n)$.

1. There is a bound, depending only on $m$ and $n$, for the number of edges of $\Gamma$.

2. If $m \neq n$, and $G \neq K$, every prime $p$ dividing a label of $\Gamma$ must divide $m n$.

Proof. This follows easily from Theorem 4.1, noting that at most 2 labels of $\Gamma$ may be equal to \pm 1 . If $\Gamma$ is a lollipop, $(m, n)$ is a multiple of $(Q X, Q Y)$ or $(Q Y, Q X)$. In particular $Q, X, Y$ are bounded, so the number of edges is bounded. Primes dividing $Q, X$ or $Y$ divide $m$ or $n$, and primes dividing $R$ divide $X$ or $Y$ by Lemma 2.5, so Assertion 2 holds in this case. If $\Gamma$ is a segment, then $m=n$ and the number of edges is bounded because $Q$ or $R$ divides $m$.

Remark 5.2. In the segment case, one of the numbers $Q$ or $R$ is bounded in terms of $m, n$, but there is no control on the other. In the lollipop case, $Q, X, Y$ are bounded, but $R$ does not have to be. See also Remark 5.8.

Proposition 5.3. Given $m$ and $n$, the following are equivalent (up to swapping $m$ and $n)$ :

1. every non-solvable $G B S$ quotient of $B S(m, n)$ is isomorphic to $B S(m, n)$;

2. $m= \pm 1$, or $|m|$ is a prime number and $m \neq n$.

Proof. 2 implies 1 by Theorem 4.1 and Lemma 2.8. We now construct a non-solvable quotient under the assumption that 2 does not hold. In particular, $m, n \neq \pm 1$. If $m=n$, we map $B S(m, n)$ to $\left\langle a, b \mid a^{m}=b^{N}\right\rangle$ with $N$ arbitrary, so assume $m \neq n$. Then none of $|m|,|n|$ is prime. If $p$ is a prime dividing $m$ and $n$, then $B S(m, n)$ maps onto $B S(m / p, n / p)$ (see Lemma 3.5). If $m, n$ are coprime, we write $m=\alpha \beta$ and $n=\gamma \delta$ with integers $\alpha, \beta, \gamma, \delta$ different from \pm 1 , and we consider $G=\left\langle a, b, t \mid a^{\alpha}=b^{\gamma}, t b^{\beta} t^{-1}=a^{\delta}\right\rangle$ (it is represented by a circle of length 2). It is generated by $a$ and $t$, which satisfy $t a^{\alpha \beta} t^{-1}=a^{\gamma \delta}$, so it is a quotient of $B S(m, n)$. It is not isomorphic to $B S(m, n)$ by [8] since $\alpha \wedge \delta=\beta \wedge \gamma=1$ ( $\Gamma$ is strongly slide-free).

Corollary 5.4. Given $m$ and $n$, the following are equivalent (up to swapping $m$ and $n$ ):

1. every non-cyclic $G B S$ quotient of $B S(m, n)$ is isomorphic to $B S(m, n)$;

2. $m= \pm 1$, or $|m|$ is a prime number not dividing $n$.

Proof. As before, 2 implies 1 by Theorem 4.1 and Lemma 2.8. For the converse, we simply observe that $B S(m, n)$ maps onto $B S\left(1, \frac{n}{m}\right)$ if $|m|$ is a prime dividing $n$.

Corollary 5.5. If $G$ is a Baumslag-Solitar group, the following are equivalent:

- every epimorphism from $G$ to a non-cyclic GBS group is an isomorphism;

- $G$ is a solvable $B S(1, n)$. 
This is a special case of Theorem A of [6].

Proof. This follows from the previous corollary: $B S(1, n)$ is Hopfian, but $B S(m, n)$ is not when $m$ is a prime not dividing $n \neq \pm 1$ (see [2, 5] and Remark 6.10).

Proposition 5.6. Given $m$ and $n$, the following are equivalent:

1. if a $G B S$ quotient $G$ of $B S(m, n)$ is represented by a labelled graph $\Gamma$ with no label \pm 1 , and $G \neq K$, then $\Gamma$ is homeomorphic to a circle;

2. up to isomorphism, there are only finitely many $G B S$ quotients of $B S(m, n)$;

3. one of the following holds (up to swapping $m$ and $n$ ):

(a) $m$ and $n$ are coprime;

(b) $|m|$ is prime and $m \neq n$;

(c) $m=-n$;

(d) $|m|$ and $|n|$ are powers of the same prime $p$, and $m \neq n$.

In particular:

Corollary 5.7. If $B S(m, n)$ has a $G B S$ quotient $G \neq K$ represented by a labelled graph $\Gamma$ with no label equal to \pm 1 such that $\Gamma$ is not homeomorphic to a circle, then $B S(m, n)$ has infinitely many non-isomorphic GBS quotients.

Remark 5.8. When none of $m, n$ divides the other, reduced labelled graphs representing a quotient of $B S(m, n)$ have no label \pm 1 , and there are finitely many such graphs. But when divisibility occurs it is important in the proposition and the corollary to exclude labels equal to \pm 1 . For instance, $B S(2,4)$ has finitely many GBS quotients up to isomorphism, but it is represented by the (reduced) non-circle lollipop of Figure 2 with $R$ an arbitrarily large power of 2 (and $Q=Y=2, X=1$ ).

Proof of Proposition 5.6. Lemma 2.8 and Remark 5.2 show that 1 implies 2. We show that 3 implies 1 . Segments are ruled out since each assumption implies $m \neq n$ (unless $m=n= \pm 1$ ). Non-circle lollipops are ruled out under the first two assumptions. Under the other two, $X$ and $Y$ are opposite or powers of the same prime (up to sign), so lollipops are ruled out by the last assertion of Lemma 2.5.

To show that 2 implies 3, we assume that none of the conditions (a)-(d) holds, and we construct infinitely many quotients. To prove that they are not isomorphic, we use a result by Clay-Forester [4] (see Section 2.2 of [3] for the case of GBS groups): if two reduced labelled graphs representing non-elementary GBS groups cannot be connected by a finite sequence of admissible sign changes, slide moves, induction moves, $\mathcal{A}^{ \pm 1}$ moves, they represent non-isomorphic groups.

We distinguish three cases.

If $m=n \neq \pm 1$, then $B S(m, m)$ maps onto $\left\langle a, b \mid a^{m}=b^{N}\right\rangle$ for any $N$.

If none of $m, n$ divides the other, we write $m=\delta m^{\prime}$ and $n=\delta n^{\prime}$, with $m^{\prime}, n^{\prime}$ coprime and $\delta, m^{\prime}, n^{\prime} \neq \pm 1$. Let $p$ be a prime dividing $n^{\prime}$. For $N>1$ such that $p^{N}$ does not divide $n^{\prime}$, consider

$$
G_{N}=\left\langle a, b, t \mid a^{\delta}=b^{p^{N}}, t b^{m^{\prime}} t^{-1}=b^{n^{\prime}}\right\rangle
$$

as on Figure 2, These groups are quotients of $B S(m, n)$ by the easy direction of Theorem 4.1. Using [4], one checks that they are pairwise non-isomorphic: no sequence of moves can transform the graph defining $G_{N}$ into the graph defining $G_{M}$ for $M \neq N$.

If $m$ divides $n$, and $m \neq n$, we may write $m=\alpha \beta$ and $n=\alpha \beta \gamma \delta$ with $\alpha, \delta$ coprime and $\alpha, \beta, \delta \neq \pm 1$ (but $\gamma= \pm 1$ is allowed). We define

$$
H_{N}=\left\langle a, b, t \mid a^{\beta}=b^{\delta^{N}}, t b^{\alpha} t^{-1}=b^{\alpha \gamma \delta}\right\rangle
$$


for $N>1$ such that $\delta^{N}$ does not divide $\gamma \delta$. These groups are pairwise non-isomorphic by [4].

\section{Groups epi-equivalent to a Baumslag-Solitar group}

In Section 4 we have determined for which values of $m, n$ a given 2-generated GBS group $G$ is a quotient of $B S(m, n)$. We now consider Baumslag-Solitar quotients of $G$. Lemma 3.6. says that we should restrict to groups represented by a lollipop (possibly a circle). In this case Theorem 4.1 implies that there is a "smallest" Baumslag-Solitar group mapping to $G$, namely $B S(Q X, Q Y)$, and we shall determine whether $G$ maps onto $B S(Q X, Q Y)$ or not (Theorem 6.6). In other words, we shall find the GBS groups epi-equivalent to a given $B S(m, n)$, in the following sense.

Definition 6.1. Two groups are epi-equivalent if each is isomorphic to a quotient of the other.

Of course, any group epi-equivalent to a Hopfian group is isomorphic to it. Recall [5] that $B S(m, n)$ is Hopfian if and only if $m$ and $n$ have the same prime divisors, or one of them equals \pm 1 (see Remark 6.10).

Remark 6.2. When a 2-generated GBS group $G$ represented by a lollipop does not map onto $B S(Q X, Q Y)$ (in particular when $B S(Q X, Q Y)$ is Hopfian), there does not always exist a maximal Baumslag-Solitar quotient. For instance, $\left\langle a, b, t \mid a^{3}=b^{3}, t b^{2} t^{-1}=a^{4}\right\rangle$ is a quotient of the Hopfian group $B S(12,6)$. It maps onto $B S(4,2)$ and $B S(6,3)$, but not onto $B S(12,6)$.

We start with a simple case.

Proposition 6.3. Fix coprime integers $m$ and $n$. Given a non-cyclic GBS group $G$, the following are equivalent:

1. $G$ is a quotient of $B S(m, n)$;

2. $G$ is epi-equivalent to $B S(m, n)$;

3. $G$ may be represented by a reduced labelled graph $\Gamma$ homeomorphic to a circle, with $X=m$ and $Y=n$.

Up to isomorphism, there are only finitely many groups $G$ satisfying these conditions.

Proof. Clearly 2 implies 1. It follows from Theorem 4.1 that 1 implies 3 . If 3 holds, $G$ is 2-generated by Theorem 1.1 of 13 (there is no proper plateau in $\Gamma$ ), and is a quotient of $B S(m, n)$ by the easy direction of Theorem 4.1. Lemma 3.6 yields an epimorphism from $G$ to some Baumslag-Solitar group. Since $m$ and $n$ are coprime, this group must be $B S(m, n)$ by Lemma 3.5, so 2 holds. The finiteness statement follows from Proposition [5.6.

Remark 6.4. When $m$ and $n$ are not coprime, and $B S(m, n)$ is not Hopfian, Corollary 6.8 will show that there exist infinitely many groups epi-equivalent to $B S(m, n)$, provided none of $|m|,|n|$ is prime.

Corollary 6.5. A GBS group $G$ is large (some finite index subgroup has a nonabelian free quotient) if and only if $G$ is not a quotient of $B S(m, n)$ with $m, n$ coprime.

Proof. Groups satisfying the third condition of Proposition 6.3 with $m$ and $n$ coprime are those which may be represented by a circle containing no proper plateau, so the corollary follows from Theorem 6.7 of [13] (see also [14]). 
Theorem 6.6. A 2-generated GBS group $G$ represented by a reduced labelled graph $\Gamma$ homeomorphic to a lollipop (possibly a circle) maps onto $B S(Q X, Q Y)$ if and only if $q_{i} \wedge r_{j}=1$ for $0 \leq i<j<k$, and one of the following holds:

- $X$ and $Q Y$ are coprime;

- $Y$ and $Q X$ are coprime;

- $Q$ and $r_{k}$ are coprime, and there exists $i_{0} \in\{0, \ldots, \ell-1\}$ such that, if a prime $p$ divides both $Q X$ and $Q Y$, then it divides no $x_{i}$ with $i>i_{0}$ and no $y_{j}$ with $j \leq i_{0}$.

Note that $q_{i} \wedge r_{j}=1$ is required only for $j<k$; in particular, there is no condition when $k \leq 1$. Also note that $i_{0}$ always exists when $\ell=1$.

We derive two corollaries before giving the proof.

Corollary 6.7. A GBS group is epi-equivalent to a Baumslag-Solitar group if and only if it has rank 2 and may be represented by a graph $\Gamma$ satisfying the conditions of Theorem 6.6 .

Proof. Suppose that $G$ is epi-equivalent to $B S(m, n)$ and is represented by a reduced labelled graph $\Gamma$. Then $G$ has rank 2 , and $\Gamma$ is a lollipop by Lemma 3.6 (it cannot be a segment). Moreover $B S(m, n)$ is isomorphic to $B S(Q X, Q Y)$ by Theorem 4.1, and $\Gamma$ satisfies the conditions of Theorem 6.6. Conversely, $G$ is epi-equivalent to $B S(Q X, Q Y)$ if $\Gamma$ is as in the theorem.

Corollary 6.8. Suppose that $B S(m, n)$ is not Hopfian. There exist infinitely many pairwise non-isomorphic GBS groups epi-equivalent to $B S(m, n)$ if and only if $|m|$ is not prime, $|n|$ is not prime, and $m \wedge n \neq 1$.

Proof. If $m$ or $n$ is prime, or if $m, n$ are coprime, $B S(m, n)$ has finitely many GBS quotients up to isomorphism by Proposition 5.6. Otherwise, non-Hopficity implies that $m$ and $n$ do not have the same prime divisors [5]. We may assume that there is a prime $p$ dividing $n$ but not $m$. Since $m \neq \pm n$, and $|m|,|n|$ cannot be powers of the same prime, $B S(m, n)$ has infinitely many quotients $G_{N}$ or $H_{N}$ constructed in the proof of Proposition [5.6. By the third case of Theorem 6.6 ( $Q$ and $r_{k}$ coprime), the groups $G_{N}$ are epi-equivalent to $B S(m, n)$ if we construct them using $p$ as above (not dividing $m$ ). The groups $H_{N}$ are epi-equivalent to $B S(m, n)$ if we choose $\delta=p$, since $\beta$ and $\delta$ are then coprime.

The remainder of this section is devoted to the proof of Theorem 6.6.

\subsection{Contraction and displacement moves}

We first describe two general ways of constructing GBS quotients $G^{\prime}$ of a GBS group $G$ (see Figures 5 and 7 ). Let $\Gamma$ be a labelled graph representing $G$.
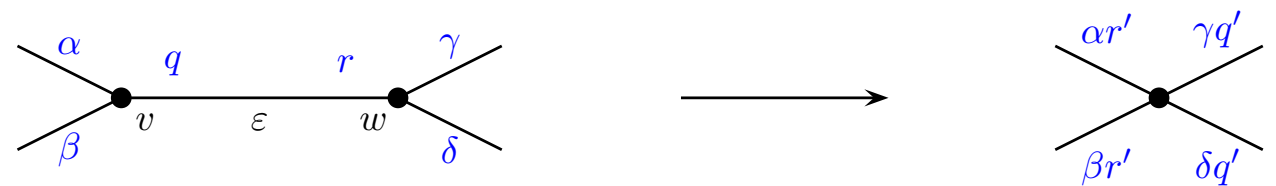

Figure 5: contraction move 
Definition 6.9 (Contraction move [6]). Consider an edge $\varepsilon=v w$ of $\Gamma$ with labels $q$ and $r$ (see Figure 5). The group associated to $\varepsilon$ is $H=\left\langle a, b \mid a^{q}=b^{r}\right\rangle$. We may map it onto $\mathbb{Z}$ by sending a to $\frac{r}{q \wedge r}$ and $b$ to $\frac{q}{q \wedge r}$ (this amounts to making $H$ abelian, and dividing by torsion if $q \wedge r>1)$. Being injective on $\langle a\rangle$ and on $\langle b\rangle$, this map extends to an epimorphism from $G$ to a GBS group $G^{\prime}$. In the process the edge $\varepsilon$ gets contracted to a point; labels near $v$ get multiplied by $r^{\prime}=\frac{r}{q \wedge r}$, labels near $w$ by $q^{\prime}=\frac{q}{q \wedge r}$.

Remark 6.10. When $q$ or $r$ equals \pm 1 , this is an elementary collapse [8] which does not change $G$ (see Figure 3). But if none of $q, r$ equals \pm 1 , the epimorphism $G \rightarrow G^{\prime}$ produced by a contraction move is not an isomorphism because $H$ is not cyclic. This gives a simple way of showing the non-Hopficity of Baumslag-Solitar groups (see Figure 6 ). If $B S(m, n)$ is not Hopfian, then (up to swapping $m$ and $n) n \neq \pm 1$ and we can write $m=p m^{\prime}$ with $p$ a prime not dividing $n$. Collapsing the edge $e$ of Figure 6 induces a group isomorphism $\varphi_{1}$, while contracting $e^{\prime}$ induces a non-injective epimorphism $\varphi_{2}$.

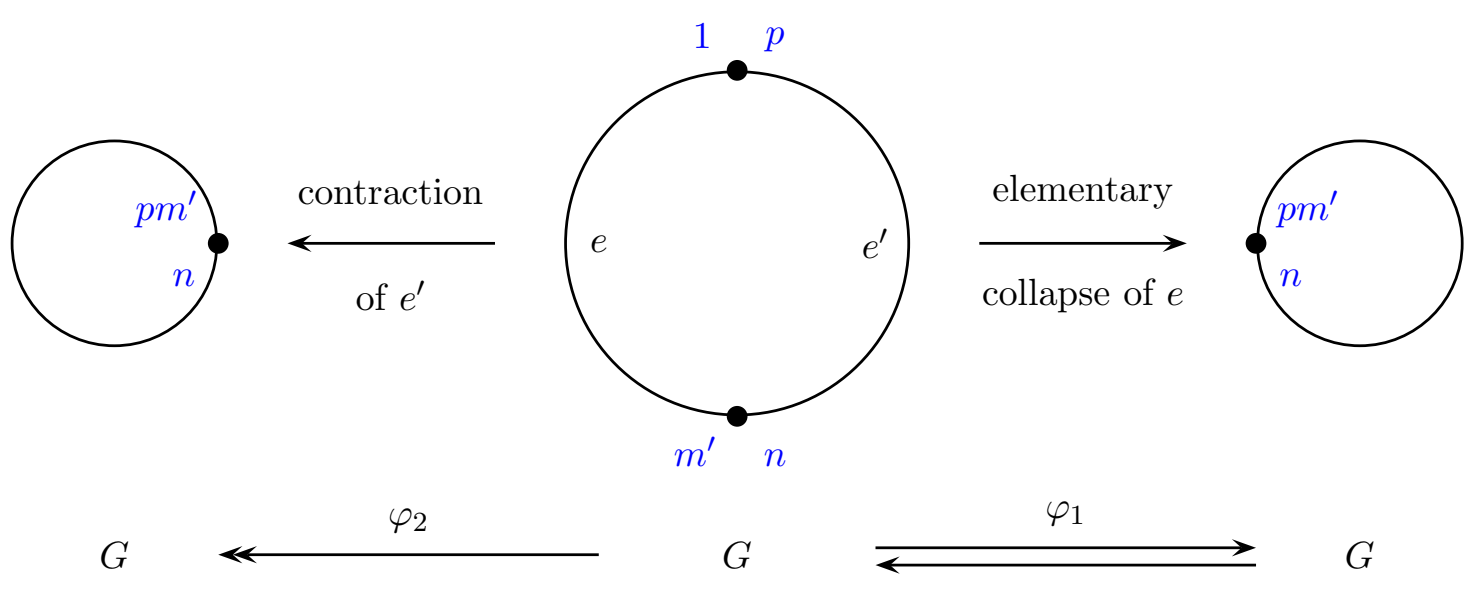

Figure 6: non-Hopficity of $B S\left(\mathrm{pm}^{\prime}, n\right)$ when $p, n$ are $>1$ and coprime; $\varphi_{1}$ is an isomorphism, $\varphi_{2}$ is not

Definition 6.11 (Displacement move). Consider an edge $\varepsilon=v w$ with labels $q$ and $r$ s (see Figure 7). We replace it by two edges $\varepsilon^{\prime}$ and $\varepsilon^{\prime \prime}$, with labels $q, r$ and $1, s$ respectively (this is the reverse of an elementary collapse, it does not change $G$ ). We then contract the edge $\varepsilon^{\prime}$. If $q$ and $r$ are coprime, the construction simply "moves" $r$ : the label of $\varepsilon$ near $w$ is divided by $r$, and labels of edges incident to $v$ are multiplied by $r$.

\subsection{The case of a circle}

We prove Theorem 6.6 when $\Gamma$ is a circle. We rephrase it as follows:

Proposition 6.12. A 2-generated GBS group $G$ represented by a reduced labelled graph $\Gamma$ homeomorphic to a circle maps onto $B S(X, Y)$ if and only if there exists $i_{0} \in\{0, \ldots, \ell-1\}$ such that, if a prime $p$ divides both $X$ and $Y$, then it divides no $x_{i}$ with $i>i_{0}$ and no $y_{j}$ with $j \leq i_{0}$.

As usual, we assume Convention 2.4 the vertex $w_{0}$ belongs to every plateau, so $G$ is generated by $b_{0}$ and $\tau$. 

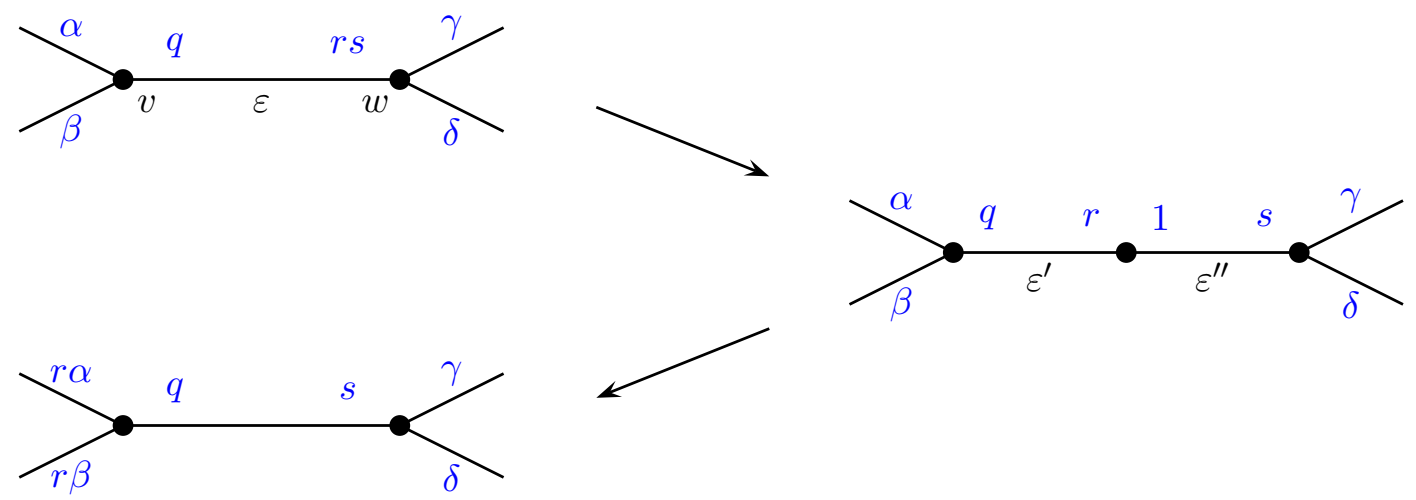

Figure 7: displacement move with $q, r$ coprime

Example 6.13. The group $G=\left\langle a, b, t \mid a^{2 \beta}=b^{2}, t b^{\gamma} t^{-1}=a^{2 \alpha}\right\rangle$ represented by $\Gamma$ (see Figure (8) is 2-generated if and only if $\gamma$ is odd, so let us assume this. The condition of the proposition holds (with $i_{0}=0$ ) if and only if no prime divisor of $X \wedge Y=2 \beta \gamma \wedge 4 \alpha$ divides $x_{1}=\gamma$; this is equivalent to $\gamma \wedge \alpha=1$.

Write $\gamma=\gamma_{1} \gamma_{2}$ with $\gamma_{1} \wedge \alpha=1$ and $\left|\gamma_{1}\right|$ maximal. The proof of the proposition will show that $G$ is epi-equivalent to the group $G^{\prime}$ represented by $\Gamma^{\prime}$; moreover, $G^{\prime}$ maps onto $B S(X, Y)=B S(2 \beta \gamma, 4 \alpha)$ if and only if $\gamma_{2}= \pm 1$, or equivalently $\gamma \wedge \alpha=1$.

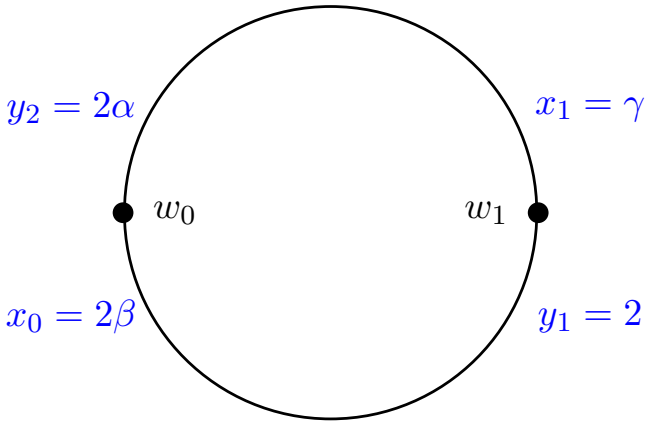

$\Gamma$

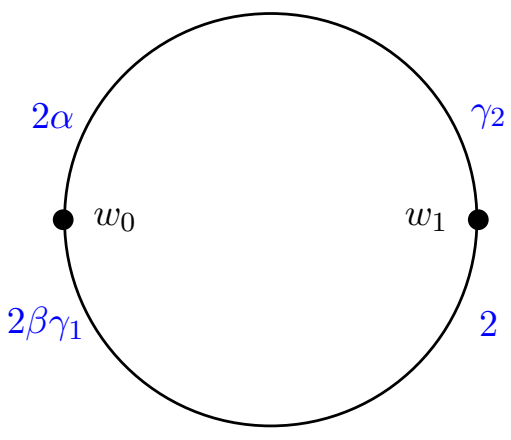

$\Gamma^{\prime}$

Figure 8: the group $G$ represented by $\Gamma$ is epi-equivalent to a Baumslag-Solitar group if and only if $\gamma$ is odd and $\gamma \wedge \alpha=1$

Proof of Proposition 6.12, We may assume that $\Gamma$ has more than one edge (i.e. $\ell>1$ ). Among the prime divisors of $X Y$, those which divide both $X$ and $Y$ will be called bilateral, the others unilateral.

If $p$ is a unilateral prime dividing some $x_{i}$ with $i>0$, a sequence of displacement moves allows us to move $p$ around the circle from $w_{i}$ to $w_{\ell}=w_{0}$ (through $w_{i+1}, \ldots, w_{\ell-1}$ ), dividing $x_{i}$ by $p$ and multiplying $x_{0}$ by $p$ (and leaving all other labels unchanged). The group represented by this new labelled graph (which may fail to be reduced) is epi-equivalent to $G$ because another sequence of displacement moves takes $p$ back to $w_{i}$ (through $w_{1}, \ldots, w_{i-1}$ ).

We iterate this construction, obtaining a labelled graph $\Gamma^{\prime}$ such that no unilateral prime divides $x_{i}$ for $i>0$, and similarly no unilateral prime divides $y_{j}$ for $j<\ell$ (for 
simplicity we write $x_{i}, y_{j}$ rather than $x_{i}^{\prime}, y_{j}^{\prime}$ for the labels carried by $\left.\Gamma^{\prime}\right)$. The group $G^{\prime}$ represented by $\Gamma^{\prime}$ is epi-equivalent to $G$.

We then make the graph reduced by collapsing edges with at least one label equal to \pm 1 (as on Figure 3). We obtain a reduced labelled graph $\Gamma^{\prime \prime}$ representing the same group $G^{\prime}$. Note that $\Gamma^{\prime}, \Gamma^{\prime \prime}$ are circles, and $X, Y$ do not change during the whole process.

If there exists $i_{0}$ as in the proposition, all labels $x_{i}$ for $i>i_{0}$ and $y_{j}$ for $j \leq i_{0}$ in the graph $\Gamma^{\prime}$ are equal to \pm 1 : they cannot be divisible by a unilateral prime because of the way $\Gamma^{\prime}$ was constructed, or by a bilateral prime because of the assumption on $i_{0}$. It follows that all edges of $\Gamma^{\prime}$, except that between $w_{i_{0}}$ and $w_{i_{0}+1}$, get collapsed in $\Gamma^{\prime \prime}$, so $G^{\prime}=B S(X, Y)$ and therefore $G$ is epi-equivalent to $B S(X, Y)$.

Conversely, we assume that $G$ (hence $G^{\prime}$ ) maps onto $B S(X, Y)$. Suppose for a moment that $\Gamma^{\prime \prime}$ consists of a single edge. Let $w_{i_{0}}$ and $w_{i_{0}+1}$ be the endpoints of the unique edge of $\Gamma^{\prime}$ which does not get collapsed in $\Gamma^{\prime \prime}$. Since the labels $x_{0}$ and $y_{\ell}$ in $\Gamma^{\prime}$ are not equal to \pm 1 , because $\Gamma$ has more than one edge, collapsibility of the other edges implies $x_{i}= \pm 1$ for $i>i_{0}$ and $y_{j}= \pm 1$ for $j \leq i_{0}$ in $\Gamma^{\prime}$. This means that the corresponding labels of $\Gamma$ cannot be divisible by a bilateral prime, so $i_{0}$ satisfies the conditions of the proposition.

We complete the proof of the proposition by showing that $G^{\prime}$ does not map onto $B S(X, Y)$ if $\Gamma^{\prime \prime}$ has more than one edge. From now on, all labels $x_{i}, y_{j}$ are those of $\Gamma^{\prime \prime}$, and $\ell>1$ is the length of $\Gamma^{\prime \prime}$. Recall that $\Gamma^{\prime \prime}$ is reduced, and no unilateral prime divides $x_{i}$ for $i>0$ or $y_{j}$ for $j<\ell$. We assume that $f: G^{\prime} \rightarrow B S(X, Y)$ is an epimorphism, and we argue towards a contradiction.

Let $p$ be a prime dividing $x_{1}$, and $p^{\prime}$ a prime dividing $y_{1}$. They are distinct because $w_{0}$ meets every plateau of $\Gamma$, and they are bilateral (they each divide both $X$ and $Y$ ). Note that $p x_{0}$ divides $X$, and $p^{\prime} y_{\ell}$ divides $Y$.

Consider the generator $b_{0}$ of the vertex group carried by $w_{0}$. Write $B S(X, Y)=\langle a, t|$ $\left.t a^{X} t^{-1}=a^{Y}\right\rangle$. The image of $b_{0}$ by $f$ is (conjugate to) a power $a^{r}$ by Lemma 3.1. By Lemma 2.6, applied to the pair $\left(f\left(b_{0}\right), f(\tau)\right)$, and Remark 2.7 the number $r$ is coprime with $X \wedge Y$, hence not divisible by either $p$ or $p^{\prime}$.

The incident edge groups at $w_{0}$ in $\Gamma^{\prime \prime}$ are generated by $\left(b_{0}\right)^{x_{0}}$ and $\left(b_{0}\right)^{y_{\ell}}$. We claim that one of them (at least) fixes a unique point in the Bass-Serre tree of $B S(X, Y)$.

Otherwise, $r x_{0}$ and $r y_{\ell}$ must each be divisible by $X$ or $Y$. Since $r x_{0}$ cannot be divisible by $X$, because $p x_{0}$ divides $X$ but not $r x_{0}$, it must be divisible by $Y$. Similarly, $X$ divides $r y_{\ell}$. Now $p^{\prime} y_{\ell}$ divides $Y$, hence $r x_{0}$, hence $r X$, hence $r^{2} y_{\ell}$, a contradiction which proves the claim.

It follows that $f$ factors through the group $\hat{G}$ obtained by contracting (in the sense of Definition 6.9) one of the edges of $\Gamma^{\prime \prime}$ adjacent to $w_{0}$, say the edge $w_{0} w_{1}$. The labels carried by this edge are $x_{0}$ and $y_{1}$, they are not coprime. Indeed, $p^{\prime}$ divides $y_{1}$ by definition; it divides $X$, and it cannot divide $x_{i}$ for $i>0$ because $w_{0}$ belongs to every plateau, so it divides $x_{0}$ (if the contracted edge is $w_{0} w_{\ell}$, we use a prime dividing $x_{\ell-1}$ to see that $x_{\ell-1}$ and $y_{\ell}$ are not coprime). It follows that the numbers $X^{\prime \prime}, Y^{\prime \prime}$ associated to $\hat{G}$ are smaller than $X$ and $Y$ (they get divided by $x_{0} \wedge y_{1}$, see Definition [6.9) , so $\hat{G}$ cannot map onto $B S(X, Y)$ by Lemma 3.5. Thus $f$ as above cannot exist.

Corollary 6.14. Let $G$ be a 2-generated GBS group represented by a reduced labelled graph $\Gamma$ homeomorphic to a circle. If there is at most one prime $p$ dividing both $X$ and $Y$, then $G$ is epi-equivalent to $B S(X, Y)$.

Proof. The result is clear if $X$ and $Y$ are coprime (see also Proposition 6.3). Otherwise, let $i_{0}$ be the largest $i$ such that $p$ divides $x_{i}$. Since $w_{0}$ belongs to every plateau, $p$ cannot divide $y_{j}$ for $j \leq i_{0}$, so $i_{0}$ is as in the proposition. 


\subsection{The general case}

We can now prove Theorem [6.6 in full generality. It follows from Proposition 6.12 if $\Gamma$ is a circle.

- We next consider the case when $k=\ell=1$ ( $\Gamma$ is a lollipop consisting of only two edges, as in Figure 21). The statement in this case is that $G$ maps onto $B S(Q X, Q Y)$ if and only if one of the numbers $X \wedge Q Y, Y \wedge Q X$, or $Q \wedge R$ equals 1.

Write

$$
G=\left\langle a_{0}, b_{0}, \tau \mid a_{0}^{Q}=b_{0}^{R}, \tau b_{0}^{X} \tau^{-1}=b_{0}^{Y}\right\rangle
$$

and

$$
B S(Q X, Q Y)=\left\langle a, t \mid t a^{Q X} t^{-1}=a^{Q Y}\right\rangle .
$$

Every prime divisor of $R$ divides $X Y$ (see Lemma 2.5), so we may write $R \tilde{R}=X^{\alpha} Y^{\beta}$ with $\tilde{R} \in \mathbb{Z}$ and $\alpha, \beta \geq 0$.

Sending $a_{0}$ to $a^{Y^{\alpha+\beta}}, b_{0}$ to $t^{\alpha} a^{\tilde{R} Q} t^{-\alpha}$, and $\tau$ to $t$ defines a homomorphism $f$ from $G$ to $B S(Q X, Q Y)$, because the relations of $G$ are satisfied:

$$
\begin{gathered}
f\left(b_{0}^{R}\right)=t^{\alpha} a^{\tilde{R} Q R} t^{-\alpha}=t^{\alpha} a^{Q X^{\alpha} Y^{\beta}} t^{-\alpha}=a^{Q Y^{\alpha+\beta}}=f\left(a_{0}^{Q}\right) \\
f\left(\tau b_{0}^{X} \tau^{-1}\right)=t t^{\alpha} a^{\tilde{R} Q X} t^{-\alpha} t^{-1}=t^{\alpha} a^{\tilde{R} Q Y} t^{-\alpha}=f\left(b_{0}^{Y}\right) .
\end{gathered}
$$

The image of $f$ contains $t$ and $a^{Y^{\alpha+\beta}}$, so it contains $a^{Q X^{\alpha+\beta}}$. If $Y \wedge Q X=1$, the map $f$ is onto, so $G$ maps onto $B S(Q X, Q Y)$. By symmetry, this is also true if $X \wedge Q Y=1$.

Now assume $Y \wedge Q X \neq 1$ and $X \wedge Q Y \neq 1$. If $f: G \rightarrow B S(Q X, Q Y)$ is an epimorphism, $f\left(a_{0}\right)$ is (conjugate to) $a^{r_{0}}$ with $r_{0}$ coprime with $Q$ and $X \wedge Y$ by Lemma 2.6 and Remark 2.7 (being 2-generated, $G$ is generated by $a_{0}$ and $\tau$ ). In particular, $f\left(a_{0}\right)$ fixes a single point in the Bass-Serre tree of $B S(Q X, Q Y)$ since $Q \neq \pm 1$. We claim that $f\left(a_{0}^{Q}\right)$ also fixes a unique point: otherwise $a^{r_{0} Q}$ would be a power of $a^{Q X}$ or $a^{Q Y}$, so $r_{0}$ would be a multiple of $X$ or $Y$; since $r_{0}$ is coprime with $Q$ and $X \wedge Y$, this contradicts $X \wedge Q Y \neq 1$ or $Y \wedge Q X \neq 1$.

It follows that any epimorphism from $G$ to $B S(Q X, Q Y)$ factors through the quotient group $\hat{G}$ obtained by contracting the edge $v_{0} w_{0}$. The labels carried by that edge are $Q$ and $R$. If they are coprime, then $\hat{G}=B S(Q X, Q Y)$ and $G$ maps onto $B S(Q X, Q Y)$. If $\delta=Q \wedge R>1$, then $\hat{G}$ is isomorphic to $B S(Q X / \delta, Q Y / \delta)$, and $G$ does not map onto $B S(Q X, Q Y)$ since $\hat{G}$ does not. This proves the theorem when $k=\ell=1$.

- The next case is when $k=1$ and $\ell$ is arbitrary. First assume $Y \wedge Q X=1$ or $X \wedge Q Y=1$. In particular, $X$ and $Y$ are coprime. As in the proof of Proposition 6.12, we may use displacement moves to construct a quotient $G^{\prime}$ of $G$ represented by a lollipop $\Gamma^{\prime \prime}$ with $k=\ell=1$, without changing $Q, X$ or $Y$ : we make all labels $x_{i}$ for $i>0$ and $y_{j}$ for $j<\ell$ equal to \pm 1 , and we collapse edges. In this process $x_{0}$ becomes $X$ and $y_{\ell}$ becomes $Y$ (the new phenomenon is that $R$ gets multiplied by some number dividing $X Y$, so we do not claim that $G^{\prime}$ is epi-equivalent to $\left.G\right)$. Since $G^{\prime}$ maps onto $B S(Q X, Q Y)$ by the previous case $(\ell=1)$, so does $G$.

If $Y \wedge Q X \neq 1$ and $X \wedge Q Y \neq 1$, we argue as in the case $\ell=1$. Any epimorphism from $G$ to $B S(Q X, Q Y)$ factors through the group $\hat{G}$ obtained by contracting the edge $v_{0} w_{0}$. This group is represented by the graph $\hat{\Gamma}$ obtained from $\Gamma$ by deleting the edge $v_{0} w_{0}$ and multiplying the labels $x_{0}$ and $y_{\ell}$ by $Q / \delta$, with $\delta=Q \wedge R$. By Proposition 6.12, it maps onto $B S(Q X, Q Y)$ if and only if $\delta=1$ and there exists $i_{0}$ as in the statement of the theorem.

- Finally, suppose $k>1$. As usual, any epimorphism $f: G \rightarrow B S(Q X, Q Y)$ maps $a_{0}$ to (a conjugate of) $a^{r_{0}}$ with $r_{0}$ coprime with $Q$ and $X \wedge Y$. Now $f\left(a_{0}^{q_{0}}\right)$ fixes a unique point in the Bass-Serre tree of $B S(Q X, Q Y)$ : otherwise $a^{r_{0} q_{0}}$ would be a power of $a^{Q X}$ or $a^{Q Y}$, so $q_{1} \cdots q_{k-1}$ would divide $r_{0}$, a contradiction (note that this argument requires 
$k \geq 2$ ). Thus $f$ factors through the group $\hat{G}$ obtained by contracting the edge $v_{0} v_{1}$. This contraction deletes the edge and multiplies $q_{1}$ by $\frac{q_{0}}{q_{0} \wedge r_{1}}$, so $G$ maps onto $B S(Q X, Q Y)$ if and only if $q_{0}, r_{1}$ are coprime and $\hat{G}$ does. The theorem now follows by induction on $k$.

\section{Baumslag-Solitar subgroups}

In this section we use immersions of graphs of groups (represented by maps between labelled graphs) to find Baumslag-Solitar subgroups in GBS groups. We show that one can characterize the set of moduli of a GBS group $G$ in terms of Baumslag-Solitar subgroups (Proposition 7.5), and we deduce that $G$ is residually finite if and only if it is solvable or virtually $F_{n} \times \mathbb{Z}$. In the last subsection we determine which Baumslag-Solitar groups $B S(r, s)$ may be embedded into a given $B S(m, n)$.

\subsection{Weakly admissible maps}

Let $G$ be a GBS group represented by a labelled graph $\Gamma$. If $\bar{G} \subset G$ is finitely generated and not free, it acts on the Bass-Serre tree of $\Gamma$ with infinite cyclic stabilizers (see Lemma 2.7 of [9]), and this yields a finite labelled graph $\bar{\Gamma}$ representing $\bar{G}$, with a map $\pi: \bar{\Gamma} \rightarrow \Gamma$ sending vertex to vertex and edge to edge (we call such a $\pi$ a morphism).

Conversely, one may use certain morphisms to construct subgroups of a given $G$. In [13] we defined and used "admissible" maps in order to represent finite index subgroups of GBS groups. We now weaken the definition in order to represent arbitrary subgroups.

Definition 7.1 (weakly admissible map). Let $\bar{\Gamma}$ and $\Gamma$ be labelled graphs. A weakly admissible map from $\bar{\Gamma}$ to $\Gamma$ is a pair $(\pi, m)$ where $\pi: \bar{\Gamma} \rightarrow \Gamma$ is a morphism, and $m$ assigns a positive multiplicity to each vertex and edge of $\bar{\Gamma}$ so that the following condition is satisfied (see Figure 9): given an edge e of $\Gamma$, with origin $v$ and label $\lambda_{e}$ near $v$, and $x \in \pi^{-1}(v)$, define $k_{x, e}$ as the gcd $m_{x} \wedge \lambda_{e}$; then there are at most $k_{x, e}$ edges of $\bar{\Gamma}$ with origin $x$ mapping to $e$, they each have multiplicity $m_{x} / k_{x, e}$, and their label near $x$ is $\lambda_{e} / k_{x, e}$.

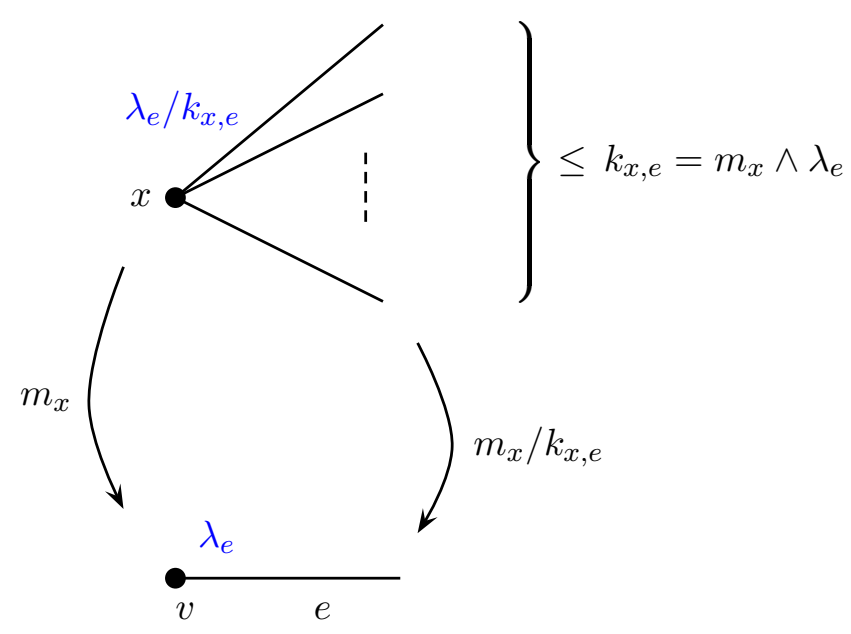

Figure 9: weakly admissible map 
The only difference between admissible ([13], Definition 6.1) and weakly admissible is the insertion of the words "at most".

We usually denote a weakly admissible map simply by $\pi$, keeping $m$ implicit.

Lemma 7.2. If $\pi: \bar{\Gamma} \rightarrow \Gamma$ is a weakly admissible map between connected labelled graphs, the GBS group $\bar{G}$ represented by $\bar{\Gamma}$ embeds into the $G B S$ group $G$ represented by $\Gamma$.

Proof. The quickest way of proving this is to show that $\pi$ defines an immersion of graphs of groups in the sense of [1] (it is a covering in the sense of [1] if and only if $\pi$ is admissible). With the notations of [1], the homomorphisms $\Phi_{a}: \mathcal{A}_{a} \rightarrow \mathcal{A}_{\Phi(a)}^{\prime}$ and $\Phi_{e}: \mathcal{A}_{e} \rightarrow \mathcal{A}_{\Phi(e)}^{\prime}$ are multiplication by the corresponding multiplicity (all groups are identified with $\mathbb{Z}$ ). The commutation relation (2.2) of [1] comes from the equality $\frac{\lambda_{e}}{k_{x, e}} \cdot m_{x}=\frac{m_{x}}{k_{x, e}} \cdot \lambda_{e}$. Since $k_{x, e}=m_{x} \wedge \lambda_{e}$, multiplication by $m_{x}$ injects the set of cosets of $\mathbb{Z}$ modulo $\frac{\lambda_{e}}{k_{x, e}}$ into the set of cosets modulo $\lambda_{e}$, so $\mathcal{A}_{a / e}$ embeds into $\mathcal{A}_{a^{\prime} / f}^{\prime}$. The fact that there are at most $k_{x, e}$ lifts ensures that the maps $\Phi_{a / f}: \amalg_{e \in \Phi_{(a)}^{-1}(f)} \mathcal{A}_{a / e} \rightarrow \mathcal{A}_{a^{\prime} / f}^{\prime}$ may be made injective.

Proposition 2.7 of 11 asserts that an immersion induces an injection between the fundamental groups of the graphs of groups, so $\bar{G}$ is a subgroup of $G$.

Remark 7.3. To prove that a group $\bar{G}$ embeds into $G$, we shall represent $\bar{G}$ and $G$ by labelled graphs $\bar{\Gamma}$ and $\Gamma$ (not necessarily reduced), define a morphism $\pi: \bar{\Gamma} \rightarrow \Gamma$, and assign multiplicities to vertices of $\bar{\Gamma}$ (but not to edges). We then check weak admissibility as follows. For each oriented edge $e$ of $\Gamma$, and each $x \in \bar{\Gamma}$ mapping onto the origin of $e$, we compute $k=m_{x} \wedge \lambda_{e}$. Each oriented edge $\bar{e}$ of $\bar{\Gamma}$ with origin $x$ mapping onto $e$ must have label $\lambda_{e} / k$, and there must be at most $k$ such edges (in our constructions, there will be at most 2 edges, so we just need $k>1$ ); we also have to check that the multiplicity $m_{x} / k$ of $\bar{e}$ is the same if we compute it using its terminal point rather than its origin $x$.

\subsection{Finding Baumslag-Solitar subgroups}

Lemma 7.4. If a non-cyclic GBS group $G$ is a quotient of $B S(m, n)$, with $m$ and $n$ coprime, it contains a subgroup isomorphic to $B S(m, n)$.

This does not hold if $m$ and $n$ are not coprime: $B S(3,5)$ is a quotient de $B S(6,10)$ (see Lemma 3.5) but does not contain it (see Theorem 7.8).

Proof. By Proposition 6.3, $G$ is represented by a reduced labelled graph $\Gamma$ which is a circle with $X=m$ and $Y=n$. We use the notations of Subsection 2.2 (see Figure 1), so the circle has length $\ell$, with $X=\prod_{j=0}^{\ell-1} x_{j}$ and $Y=\prod_{j=1}^{\ell} y_{j}$. We orient $\Gamma$ counterclockwise, so edges go from $w_{i}$ to $w_{i+1}$.

Let $\bar{\Gamma}$ be the (non-reduced) labelled graph pictured on Figure 10, It is a circle consisting of $3 \ell$ edges, which we view as 3 blocks of $\ell$ edges each. Going round the circle in the counterclockwise direction starting at the vertex on the left, the first $\ell$ edges have labels $x_{0}, \ldots, x_{\ell-1}$ at their origin and 1 at their terminal point. The next $\ell$ edges have both labels equal to 1 . The last $\ell$ edges have label 1 at their origin and $y_{1}, \ldots, y_{\ell}$ at their terminal point. Elementary collapses reduce $\bar{\Gamma}$ to the graph with one vertex and one edge, and labels $\prod_{j=0}^{\ell-1} x_{j}$ and $\prod_{j=1}^{\ell} y_{j}$, so the associated group $\bar{G}$ is isomorphic to $B S(m, n)$.

We prove the lemma by describing a weakly admissible map $\pi: \bar{\Gamma} \rightarrow \Gamma$. As indicated on Figure 10 by arrows (lifting the orientation of $\Gamma$ ), the first and third blocks of $\ell$ edges wrap around $\Gamma$ in the counterclockwise direction, the middle block in the clockwise direction. The multiplicities $m_{x}$ at vertices of $\bar{\Gamma}$ are as follows (for clarity, we write them within parentheses; three of them are indicated in red on Figure 10). In the first block:

$$
(1),\left(y_{1}\right),\left(y_{1} y_{2}\right), \ldots,\left(y_{1} \cdots y_{\ell}\right) \text {. }
$$




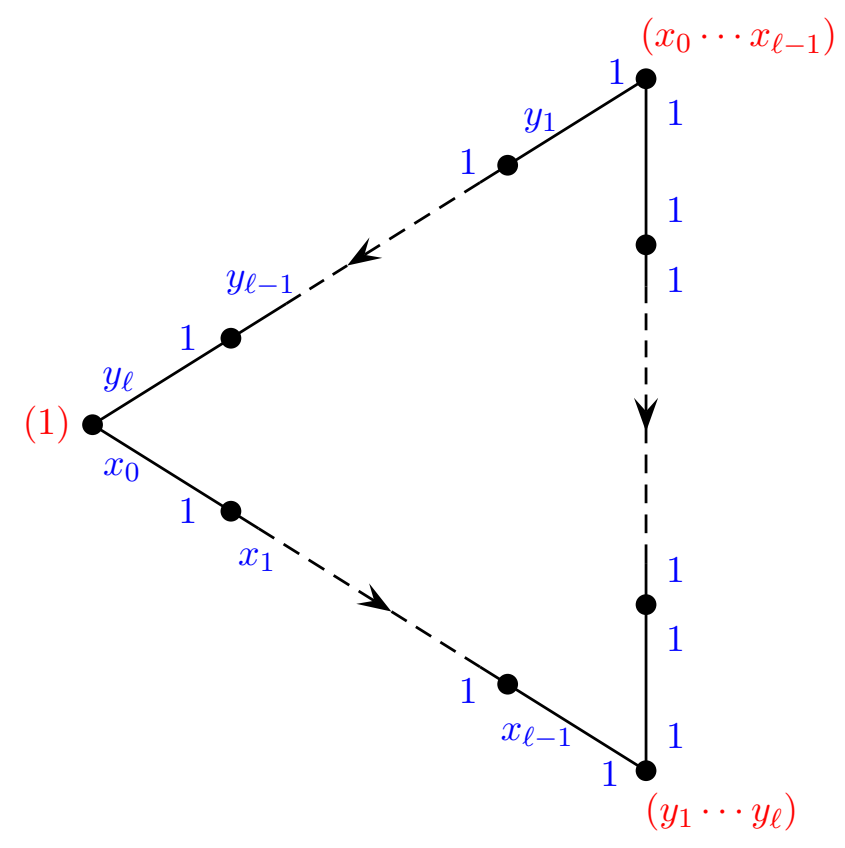

Figure 10: a non-reduced graph representing $B S(m, n)=B S(X, Y)$

In the second block:

$$
\left(y_{1} \cdots y_{\ell}\right),\left(y_{1} \cdots y_{\ell-1} x_{\ell-1}\right), \ldots,\left(y_{1} \cdots y_{i} x_{i} \cdots x_{\ell-1}\right), \ldots,\left(y_{1} x_{1} \cdots x_{\ell-1}\right),\left(x_{0} \cdots x_{\ell-1}\right) .
$$

In the third block:

$$
\left(x_{0} \cdots x_{\ell-1}\right),\left(x_{1} \cdots x_{\ell-1}\right), \ldots,\left(x_{\ell-1}\right),(1) .
$$

Weak admissibility is checked as explained in Remark 7.3, using the assumption $\prod x_{j} \wedge$ $\prod y_{j}=1$ in the first and third blocks.

Proposition 7.5. Let $r=\frac{m}{n}$ be a non-zero rational number written in lowest terms, with $r \neq \pm 1$. Let $G$ be a non-elementary GBS group. Then $r$ belongs to the image of $\Delta_{G}$ if and only if $G$ contains a subgroup $H$ isomorphic to $B S(m, n)$.

See Proposition 7.11 for the special case $r= \pm 1$.

Proof. The "if" direction is clear since a relation $t a^{m} t^{-1}=a^{n}$ with $m \neq \pm n$ implies that $a$ is elliptic, so $r=\frac{m}{n}$ is a modulus. Conversely, if $\frac{m}{n}=\Delta_{G}(t)$ and $a$ is a non-trivial elliptic element, there is a relation $t a^{q m} t^{-1}=a^{q n}$. Replacing $a$ by a power, we may assume $q=1$. The group $H=\langle a, t\rangle$ is not free, so is a non-cyclic GBS group (it acts on the Bass-Serre tree of $G$ with infinite cyclic stabilizers, see Lemma 2.7 of [9]). Being a quotient of $B S(m, n)$, the group $H$ contains $B S(m, n)$ by Lemma 7.4.

When the modulus $r$ is an integer, the proposition provides a solvable subgroup $B S(1, r)$ (see also Lemma 2.4 of [12]). If $G$ itself is not solvable, we can construct a more complicated subgroup.

Lemma 7.6. If a non-solvable GBS group $G$ contains $B S(1, n)$ with $n \neq \pm 1$, then it contains $B S(q, q n)$ for some prime $q$.

Proof. Consider the action of $G$ on the Bass-Serre tree $T$ associated to a minimal labelled graph $\Gamma$. The assumption implies that $n$ is a modulus, so let $t$ be a hyperbolic element with modulus $n$, and let $L \subset T$ be its axis. Since $G$ is not solvable, its action on $T$ is 
irreducible. It is minimal, and there is an elliptic element whose fixed point set is not the whole of $T$. By a general fact on groups acting on trees (see below), there exists an elliptic element $a$ with fixed point set disjoint from $L$. It satisfies a relation $t a^{q} t^{-1}=a^{q n}$ with $q>1$, but $t a t^{-1} \neq a^{n}$. Replacing $a$ by a power, we may ensure that these properties hold with $q$ prime. The group $H=\langle a, t\rangle$ is not solvable, so by Proposition 5.3 it is isomorphic to $B S(q, q n)$.

(To prove the fact mentioned above, use irreducibility to find $g \in G$ with $g L \cap L=\emptyset$. If the result is wrong, every elliptic element fixes the bridge between $L$ and $g L$. By minimality, the set of points fixed by all elliptic elements is $T$.)

Corollary 7.7. A GBS group is residually finite if and only if it is solvable or unimodular (virtually $F \times \mathbb{Z}$ with $F$ free).

This is proved in 15] for $G=B S(m, n)$; in this case $G$ is residually finite if and only if $m= \pm 1$, or $n= \pm 1$, or $m= \pm n$. It follows from [17] that residual finiteness is invariant under quasi-isometry among GBS groups.

Proof. A solvable GBS group is isomorphic to some $B S(1, n)$, a unimodular one is virtually $F \times \mathbb{Z}$, these groups are residually finite. Conversely, if $G$ is not unimodular and not solvable, it contains a non residually finite Baumslag-Solitar group by Proposition 7.5 if there is a modulus $r$ such that neither $r$ nor $\frac{1}{r}$ is an integer, by Lemma 7.6 otherwise.

\subsection{Embeddings of Baumslag-Solitar groups}

Theorem 7.8. Assume that $B S(r, s)$ is non-elementary, i.e. $(r, s) \neq( \pm 1, \pm 1)$. Then $B S(r, s)$ embeds into $B S(m, n)$ if and only if the following hold:

1. $\frac{r}{s}$ is a power of $\frac{m}{n}$;

2. if $m$ and $n$ are divisible by $p^{\alpha}$ but not by $p^{\alpha+1}$, with $p$ prime and $\alpha \geq 0$, then neither $r$ nor $s$ is divisible by $p^{\alpha+1}$; in particular (for $\alpha=0$ ), any prime dividing $r$ s divides mn;

3. if $m$ or $n$ equals \pm 1 , so does $r$ or $s$.

See Proposition 7.11 for the elementary case (when $B S(r, s)$ is equal to $\mathbb{Z}^{2}$ or $K$ ).

Example 7.9. Applied with $p=2$ and $\alpha=1$, the second condition implies that $B S(12,20)$ does not embed into $B S(6,10)$. It also implies that $B S(2,2)$ only contains $B S(1, \pm 1)$. On the other hand, $B S(2,3)$ contains $B S\left(2^{a+b} 3^{c}, 2^{b} 3^{a+c}\right)$ for all $a, b, c \geq 0$.

Proof. We first show that the conditions are necessary.

1 follows from Lemma 3.4: the image of $\Delta_{B S(r, s)}$ in $\mathbb{Q}^{*}$, generated by $\frac{r}{s}$, is contained in the image of $\Delta_{B S(m, n)}$, generated by $\frac{m}{n}$.

For 2, we first show that the following property holds in $B S(m, n)$ : if a is elliptic and $g$ is arbitrary, there is a relation $a^{N}=g a^{M} g^{-1}$ with $N$ not divisible by $p^{\alpha+1}$. We may assume that $a$ generates the stabilizer of a vertex $v$ in the Bass-Serre tree of $B S(m, n)$. Then $g a g^{-1}$ generates the stabilizer of $w=g v$. We apply Lemma 4.3 to the segment $v w$. All numbers $q_{j}, r_{i}$ (for $i>0$ ) are equal to $m$ or $n$. By the fourth assertion of the lemma, there is a relation $a^{N}=\left(g a g^{-1}\right)^{M}$ with $N$ not divisible by $p^{\alpha+1}$.

By Lemma 3.1, the property also holds in $B S(r, s)$. It implies that $r$ and $s$ are not divisible by $p^{\alpha+1}$.

3 is just the fact that a subgroup of a solvable group is solvable.

We shall now construct an embedding of $B S(r, s)$ into $B S(m, n)$, assuming that the three conditions are satisfied. Writing $B S(x, y)=\left\langle a_{x, y}, t_{x, y} \mid t_{x, y}\left(a_{x, y}\right)^{x} t_{x, y}^{-1}=\left(a_{x, y}\right)^{y}\right\rangle$, we say that $q$ is an index of an embedding $B S(r, s) \hookrightarrow B S(m, n)$ if $a_{r, s}$ maps to a conjugate of $\left(a_{m, n}\right)^{q}$ (a given embedding may have several indices). 
Lemma 7.10. Let $\nu$ be a non-zero integer.

1. $B S(m, n)$ embeds into $B S(\nu m, \nu n)$, with index $\nu$.

2. If there is an embedding $i: B S(r, s) \hookrightarrow B S(m, n)$ with an index $q$ coprime with $\nu$, then $B S(\nu r, \nu s)$ embeds into $B S(\nu m, \nu n)$, with an index dividing $q$.

Proof. The presentation $B S(\nu m, \nu n)=\left\langle a, b, t \mid a^{\nu}=b, t b^{m} t^{-1}=b^{n}\right\rangle$ expresses $B S(\nu m, \nu n)$ as the amalgam of $\langle a\rangle$ with $\langle b, t\rangle \simeq B S(m, n)$ over $\left\langle a^{\nu}\right\rangle=\langle b\rangle$. This proves 1 .

For 2, consider $i: B S(r, s) \hookrightarrow B S(m, n) \subset B S(\nu m, \nu n)$ with $B S(m, n)=\langle b, t\rangle$ as above and $i\left(a_{r, s}\right)=b^{q}$. First assume that none of $r, s$ is equal to \pm 1 . Then $a_{r, s}$ has no proper root in $B S(r, s)$, so $i(B S(r, s)) \cap\langle b\rangle$ is generated by $b^{q}$. Also note that $\left\langle a^{q}\right\rangle \cap\langle b\rangle$ is generated by $b^{q}=\left(a^{q}\right)^{\nu}$ because $\nu \wedge q=1$. It follows that the subgroup of $B S(\nu m, \nu n)$ generated by $i(B S(r, s))$ and $a^{q}$ is isomorphic to $B S(\nu r, \nu s)$ (we are using the fact that, given an amalgam $H=A *_{C} B$ and subgroups $A_{1} \subset A, B_{1} \subset B$ with $A_{1} \cap C=B_{1} \cap C$, the subgroup of $H$ generated by $A_{1}$ and $B_{1}$ is isomorphic to $\left.A_{1} * A_{1} \cap B_{1} B_{1}\right)$.

If $B S(r, s)$ is solvable, define $q^{\prime}$ (dividing $q$ ) by $i(B S(r, s)) \cap\langle b\rangle=\left\langle b^{q^{\prime}}\right\rangle$, and let $a_{r, s}^{\prime}=$ $i^{-1}\left(b^{q^{\prime}}\right)$. We then have $B S(r, s)=\left\langle a_{r, s}^{\prime}, t_{r, s} \mid t_{r, s}\left(a_{r, s}^{\prime}\right)^{r} t_{r, s}^{-1}=\left(a_{r, s}^{\prime}\right)^{s}\right\rangle$, and we may argue as in the previous case.

We may assume that $m$ and $n$ are different from \pm 1 (the theorem is easy otherwise). We represent $B S(m, n)$ by its standard labelled graph $\Gamma_{m, n}$, oriented so that the edge has initial label $m$ and terminal label $n$. Using Condition 1 , we write $\frac{r}{s}=\left(\frac{m}{n}\right)^{\beta}$; we may assume $\beta \geq 0$. We write $(r, s)=\left(\gamma r^{\prime}, \gamma s^{\prime}\right)$ with $r^{\prime} \wedge s^{\prime}=1$, and $(m, n)=\left(\delta m^{\prime}, \delta n^{\prime}\right)$ with $m^{\prime} \wedge n^{\prime}=1$.

We first assume that no prime appears with the same exponent $\alpha>0$ in $m$ and $n$, so every prime dividing $r s$ divides $m^{\prime} n^{\prime}$. We distinguish three subcases.

- Assume that $m \wedge n=1$. Using the first assertion of Lemma 7.10 to increase $\gamma$ if needed, we may assume $r=m^{x+\beta} n^{y}$ and $s=m^{x} n^{y+\beta}$ with $x, y \geq 1$ and $\beta \geq 0$. We represent $B S(r, s)$ by the non-reduced labelled graph $\bar{\Gamma}$ pictured on Figure 11, oriented in the counterclockwise direction. Going around the circle starting on the left, one encounters 7 blocks:

(1) $x+\beta$ edges with labels $m, 1$

(2) $x+\beta$ edges with both labels 1

(3) $y$ edges with labels $n, 1$

(4) $x+y+\beta$ edges with both labels 1

(5) $x$ edges with labels $1, m$

(6) $y+\beta$ edges with both labels 1

(7) $y+\beta$ edges with labels $1, n$.

We let $\pi$ be the unique morphism from $\bar{\Gamma}$ to $\Gamma_{m, n}$ which preserves orientation on blocks $1,4,7$ and reverses it on blocks $2,3,5,6$ (see Figure 11, with the arrows lifting the orientation of $\left.\Gamma_{m, n}\right)$.

We define multiplicities of vertices as follows (some of them are indicated within parentheses on Figure 11). On each block, they form a geometric progression; they go from 1 to $n^{x+\beta}$ on block 1 , from $n^{x+\beta}$ to $m^{x+\beta}$ on block 2 , from $m^{x+\beta}$ to $m^{x+y+\beta}$ on block 3 , from $m^{x+y+\beta}$ to $n^{x+y+\beta}$ on block 4 , from $n^{x+y+\beta}$ to $n^{y+\beta}$ on block 5 , from $n^{y+\beta}$ to $m^{y+\beta}$ on block 6 , from $m^{y+\beta}$ to 1 on block 7 .

One checks that $\pi$ is weakly admissible as explained in Remark 7.3 ( $k>1$ is guaranteed by the assumption $m, n \neq \pm 1$ ).

- Assume that $m, n$ are not coprime, but none divides the other. In this case $m^{\prime}$ and $n^{\prime}$ are different from \pm 1 , and we can write $B S(r, s) \hookrightarrow B S\left(m^{\prime}, n^{\prime}\right) \subset B S(m, n)$ using the previous case and Lemma 7.10 . 


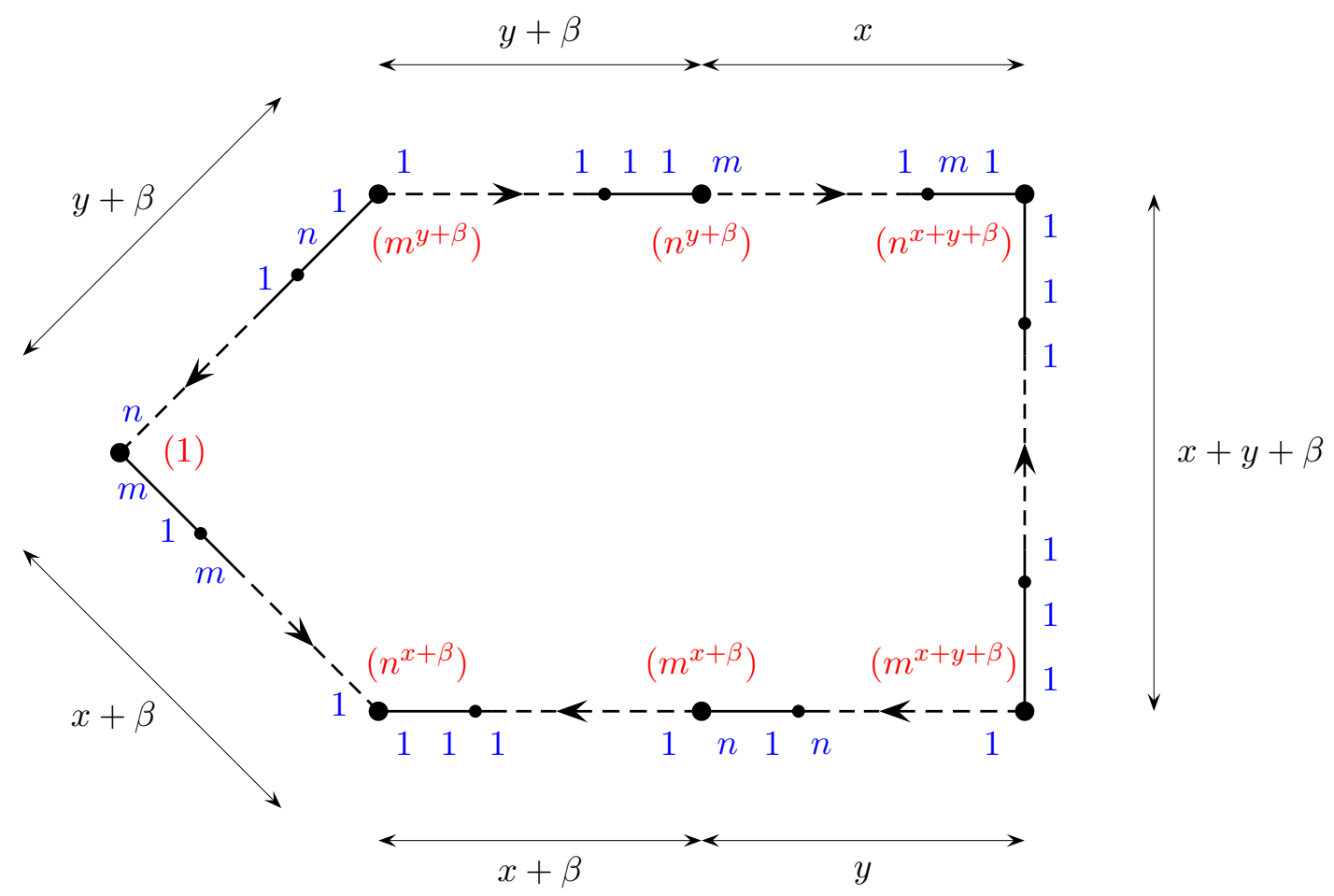

Figure 11: embedding $B S\left(m^{x+\beta} n^{y}, m^{x} n^{y+\beta}\right)$ into $B S(m, n)$ when $m \wedge n=1$

- If $m$ divides $n$, we use the fact (proved below) that $B S\left(\Delta^{x}, \Delta^{y}\right)$ embeds into $B S(m, \Delta m)$ if $m \neq \pm 1$ and $x, y \geq 1$ (and $\Delta \neq 0$ is arbitrary). We write $n=\Delta m$ and $s=\Delta^{\beta} r$. Every prime dividing $r s$ divides $\Delta$, so we may assume $r=\Delta^{x}$ with $x \geq 1$. We then have $B S(r, s)=B S\left(\Delta^{x}, \Delta^{x+\beta}\right) \hookrightarrow B S(m, \Delta m)=B S(m, n)$.

To prove the fact, we represent $B S\left(\Delta^{x}, \Delta^{y}\right)$ by a labelled graph $\Gamma$ which is a circle consisting of $x+y$ edges with labels $\Delta$ and 1 placed as on Figure 12, We map it to $\Gamma_{m, \Delta m}$ by the unique morphism $\pi$ with the following property: each edge of $\Gamma$, oriented so that the label $\Delta$ is near its origin and the label 1 near its terminal point, maps onto the edge of $\Gamma_{m, \Delta m}$ oriented with $\Delta m$ near its origin and $m$ near its terminal point (see Figure 12). We assign multiplicity $m$ to all vertices, multiplicity 1 to all edges. One easily checks that $\pi$ is weakly admissible. For future reference, we note that we may also embed $B S\left(m \Delta^{x}, m \Delta^{y}\right)$ into $B S(m, \Delta m)$, by adding to $\Gamma$ an edge with labels $m$ and 1 (the multiplicity at the terminal vertex of the new graph is $\Delta$ ).

This concludes the case when no prime appears with the same exponent in $m$ and $n$. We observe that, for the weakly admissible maps $\pi$ constructed so far, multiplicities are divisible only by prime numbers dividing $m n$. With the notations of Lemma 7.10, this implies that we constructed an embedding $B S(r, s) \hookrightarrow B S(m, n)$ with an index $q$ divisible only by primes dividing $m n$.

In the general case, we let $\delta_{1}$ be the product of the primes appearing with the same exponent in $m$ and $n$, and we write $(m, n)=\left(\delta_{1} m_{1}, \delta_{1} n_{1}\right)$. Note that $\delta_{1} \wedge m_{1} n_{1}=1$, but $m_{1}, n_{1}$ do not have to be coprime. Using Conditions 1 and 2 of the theorem, and Lemma 7.10, we may assume $(r, s)=\left(\delta_{1} r_{1}, \delta_{1} s_{1}\right)$ with $\delta_{1} \wedge r_{1} s_{1}=1$. Every prime dividing $r_{1} s_{1}$ divides $m_{1} n_{1}$.

If none of $m_{1}, n_{1}$ equals \pm 1 , the previous analysis allows us to embed $B S\left(r_{1}, s_{1}\right)$ into $B S\left(m_{1}, n_{1}\right)$ with an index coprime with $\delta_{1}$. The second assertion of Lemma 7.10 embeds $B S(r, s)=B S\left(\delta_{1} r_{1}, \delta_{1} s_{1}\right)$ into $B S\left(\delta_{1} m_{1}, \delta_{1} n_{1}\right)=B S(m, n)$.

Now suppose $m_{1}=1$, so $\delta_{1}=m \neq \pm 1$. Every prime dividing $r_{1} s_{1}$ divides $\Delta=n_{1}$, 


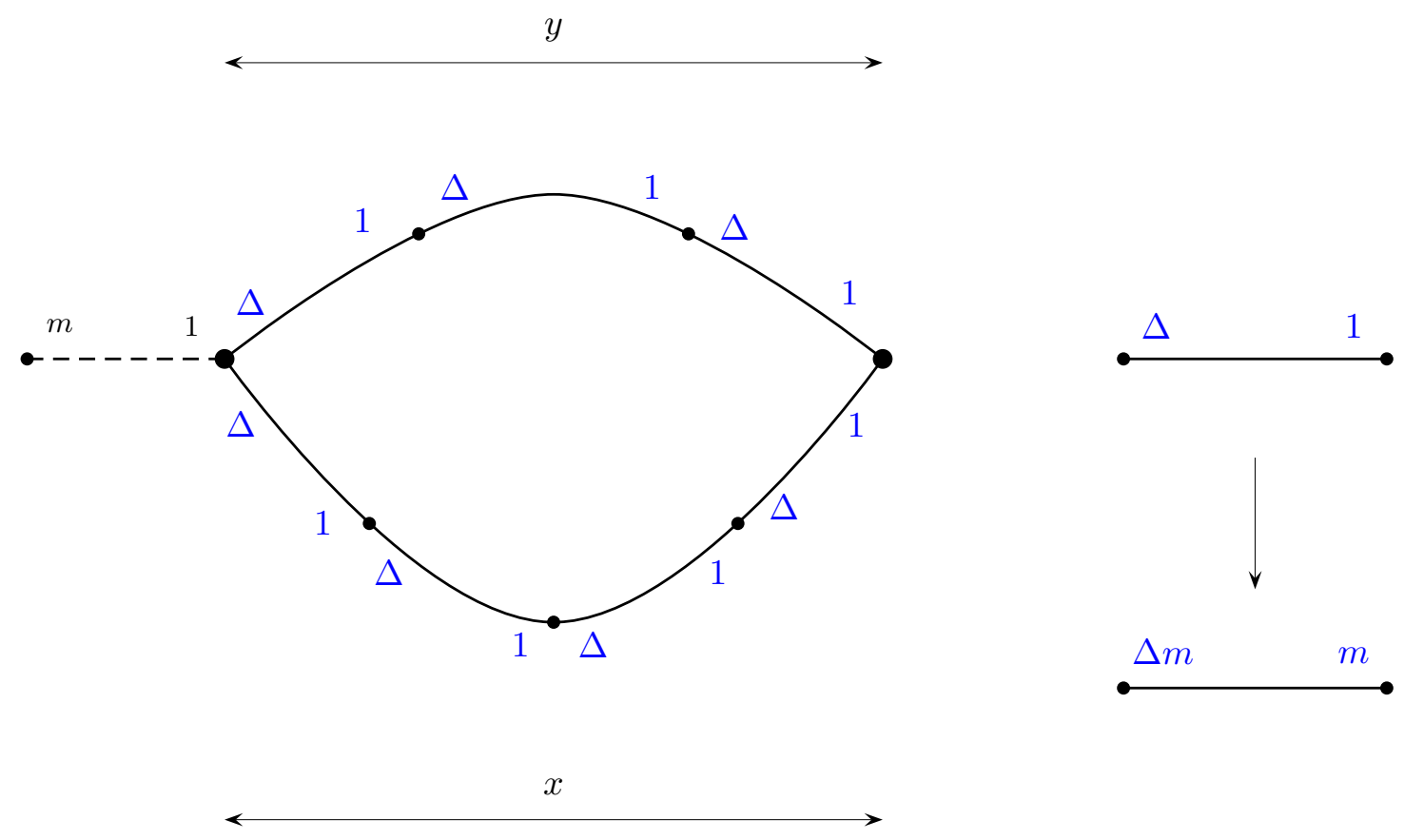

Figure 12: embedding $B S\left(\Delta^{x}, \Delta^{y}\right)$ (and $\left.B S\left(m \Delta^{x}, m \Delta^{y}\right)\right)$ into $B S(m, \Delta m)$

so (as above) we may assume $r_{1}=\Delta^{x}$ and $s_{1}=\Delta^{x+\beta}$ with $x \geq 1$. Using a fact proved earlier, we may embed $B S(r, s)=B S\left(m \Delta^{x}, m \Delta^{x+\beta}\right)$ into $B S(m, \Delta m)=B S(m, n)$.

Proposition 7.11. $\quad-\mathbb{Z}^{2}$ embeds into every Baumslag-Solitar group, except BS $(1, n)$ for $n \neq \pm 1$. The Klein bottle group $K$ embeds into $B S(m,-m)$ and $B S(2 m, n)$ for $n \neq \pm 1$, and into no other.

- More generally, every $G B S$ group other than $B S(1, n)$ contains $\mathbb{Z}^{2}$. A GBS group $G$ other than $B S(1, n)$ contains $K$ if and only if -1 is a modulus of $G$, or $G$ may be represented by a labelled graph $\Gamma$ with at least one even label.

Sketch of proof. In most cases (in particular if no label of $\Gamma$ equals \pm 1 ), one embeds $\mathbb{Z}^{2}$ or $K$ by observing that $\left\langle a, b \mid a^{m}=b^{n}\right\rangle$ contains $\mathbb{Z}^{2}$ if $m$ and $n$ are $\neq \pm 1$, and contains $K$ if in addition $m$ or $n$ is even. We leave the remaining cases to the reader. Conversely, if $G$ contains $K=\left\langle a, t \mid t a t^{-1}=a^{-1}\right\rangle$, then -1 is a modulus if $a$ is elliptic. If $a$ is hyperbolic, $t$ acts as a reflection on its axis, and this forces some label to be even.

\section{Subgroups of $B S(n, n)$}

We denote by $Z(G)$ the center of a GBS group $G$. If $G \neq \mathbb{Z}^{2}$, it is trivial or infinite cyclic.

Theorem 8.1. Given a non-cyclic GBS group $G$, and $n \geq 2$, the following are equivalent:

1. $G$ embeds into $B S(n, n)$;

2. $G / Z(G)$ is a free product of cyclic groups, with the order of each finite factor dividing $n$;

3. $G$ may be represented by a reduced labelled graph $\Gamma$ such that all labels near any given vertex $v$ are equal, and they divide $n$. 
This does not hold for $n=1$, because of $K$ and $F \times \mathbb{Z}$ (with $F$ free).

If $\Gamma$ is as in 3, so is any reduced labelled graph $\Gamma^{\prime}$ representing $G$ (up to admissible sign changes), because $\Gamma$ and $\Gamma^{\prime}$ differ by slide moves ([10], Theorem 7.4).

There is a similar characterization of groups with embed into $B S(n, \pm n)$. One must replace $Z(G)$ by an infinite cyclic normal subgroup, and allow labels near a given vertex to be opposite. In particular, $G$ embeds into $B S(n, n)$ or $B S(n,-n)$ if and only if it may be represented by a reduced labelled graph $\Gamma$ such that all labels near any given vertex $v$ are equal up to sign, and they divide $n$.

Proof. We may assume that $G$ is non-elementary. We let $\Gamma_{n, n}$ be the standard splitting of $B S(n, n)$ as an HNN extension, and we orient its edge. We denote by $T$ the associated Bass-Serre tree. The stabilizer of any edge is equal to the center of $B S(n, n)$.

If $G \subset B S(n, n)$, we consider its action on the minimal $G$-invariant subtree $T_{\min } \subset T$. Being a non-cyclic GBS group, $G$ is one-ended ([9], Lemma 2.6), so edge stabilizers are non-trivial. If $g \in G$ fixes an edge, it is central in $B S(n, n)$ hence in $G$. In particular, $Z(G)$ is infinite cyclic. The action of $G$ on $T_{\min }$ being irreducible because $G$ is non-elementary, a standard argument implies that $Z(G)$ acts as the identity on $T_{\min }$ (see [12, Proposition 2.5).

We have shown that every edge stabilizer for the action of $G$ on $T_{\min }$ is infinite cyclic and equal to $Z(G)$. For the induced action of $G / Z(G)$, edge stabilizers are trivial and vertex stabilizers are finite cyclic groups. Their order divides $n$ because they may be expressed as $G \cap\langle a\rangle / G \cap\left\langle a^{n}\right\rangle$ for some $a \in B S(n, n)$. We have proved that 1 implies 2 .

If $G$ is as in 2, we let $G / Z(G)$ act on a reduced tree $S$ with trivial edge stabilizers, and vertex stabilizers cyclic of order dividing $n$. Lift the action to $G$. Edge stabilizers are infinite cyclic (they are non-trivial by one-endedness of $G$ ). Vertex stabilizers are finite extensions of $\mathbb{Z}$, so are infinite cyclic because they are torsion-free.

Near each vertex $v$, all edge stabilizers are equal, and their index in the stabilizer of $v$ divides $n$. This means that in the labelled graph $\Gamma=S / G$ all labels near a given vertex are equal in absolute value. They are actually equal if we fix a generator $z$ of $Z(G)$ and require that all generators of vertex and edge groups of $\Gamma$ be positive roots of $z$. We have proved that 2 implies 3 .

Finally, let $G$ be as in 3. Using elementary expansions (the converse of elementary collapses), we represent $G$ by a (non-reduced) labelled graph $\Gamma^{\prime}$ such that all labels near a non-terminal vertex are equal to 1 , and these vertices are trivalent (see an example on Figure 13). It is easy to orient edges of $\Gamma^{\prime}$ so that each trivalent vertex has at least one incoming and one outgoing edge. Let then $\pi$ be the only orientation-preserving graph morphism from $\Gamma^{\prime}$ to $\Gamma_{n, n}$. We now define multiplicities so that $\pi$ is weakly admissible in the sense of Definition 7.1.
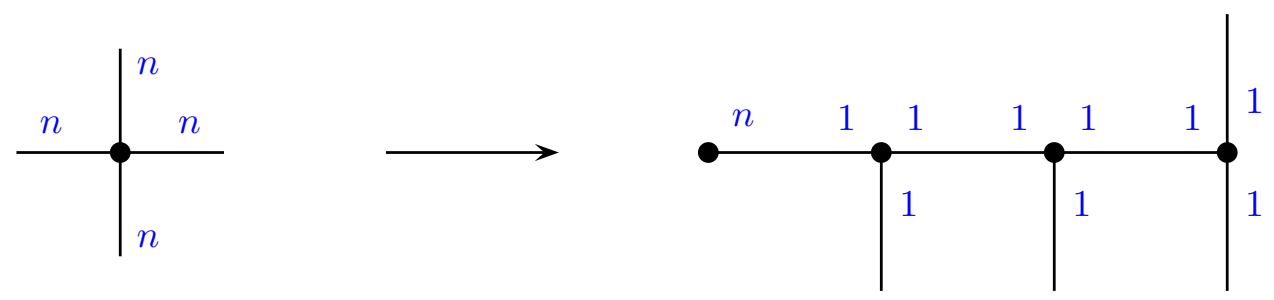

Figure 13: making non-terminal vertices trivalent with all labels 1

All edges have multiplicity 1. A terminal vertex of $\Gamma^{\prime}$ with label $d$ has multiplicity $n / d$. Trivalent vertices of $\Gamma^{\prime}$ have multiplicity $m$. The number of incoming (resp. outgoing) edges 
is at most 2, which is not bigger than $k_{x, e}$ since $k_{x, e}=n \wedge n=n$ and we assume $n \geq 2$.

Corollary 8.2. A finitely generated group $G$ embeds into some $B S(n, n)$ if and only if $G$ is torsion-free, $Z(G)$ is cyclic, and $G / Z(G)$ is a free product of cyclic groups.

Theorem 1.4 is a restatement of this corollary.

Proof. Suppose that $G$ is as in the corollary. If $Z(G)$ is trivial, then $G$ is free and embeds into any $B S(n, n)$ with $n \geq 2$. If $Z(G)$ is infinite cyclic, the proof of $2 \Rightarrow 3$ above shows that $G$ is a GBS group, so it embeds into some $B S(n, n)$ by the theorem. Conversely, any finitely generated subgroup of a GBS group is free or a GBS group, and Theorem 8.1 applies.

\section{References}

[1] H. Bass, Covering theory for graphs of groups, J. Pure Appl. Algebra 89 (1993), no. $1-2,3-47$.

[2] G. Baumslag, D. Solitar, Some two-generator one-relator non-Hopfian groups, Bull. Amer. Math. Soc. 68 (1962), 199-201.

[3] M. Clay, M. Forester, On the isomorphism problem for generalized Baumslag-Solitar groups, Algebr. Geom. Topol. 8 (2008), no. 4, 2289-2322.

[4] M. Clay, M. Forester, Whitehead moves for G-trees, Bull. Lond. Math. Soc. 41 (2009), no. 2, 205-212.

[5] D. Collins, F. Levin, Automorphisms and Hopficity of certain Baumslag-Solitar groups, Arch. Math. (Basel) 40 (1983), no. 5, 385-400.

[6] A. Delgado, D. Robinson, M. Timm, Generalized Baumslag-Solitar groups and geometric homomorphisms, J. Pure Appl. Algebra 215 (2011), no. 4, 398-410.

[7] F.A. Dudkin, Subgroups of Baumslag-Solitar groups, (Russian) Algebra Logika 48 (2009), no. 1, 3-30, 150, 152; translation in Algebra Logic 48 (2009), no. 1, 1-19.

[8] M. Forester, Deformation and rigidity of simplicial group actions on trees, Geom. Topol. 6 (2002), 219-267.

[9] M. Forester, On uniqueness of JSJ decompositions of finitely generated groups, Comment. Math. Helv. 78 (2003), no. 4, 740-751.

[10] M. Forester, Splittings of generalized Baumslag-Solitar groups, Geom. Dedicata 121 (2006), 43-59.

[11] D. Kochloukova, Injective endomorphisms of the Baumslag-Solitar group, Algebra Colloq. 13 (2006), no. 3, 525-534.

[12] G. Levitt, On the automorphism group of generalized Baumslag-Solitar groups, Geom. Topol. 11 (2007), 473-515.

[13] G. Levitt, Generalized Baumslag-Solitar groups: rank and finite index subgroups, Ann. Inst. Fourier (to appear).

[14] T. Mecham, Largeness of graphs of abelian groups, Thesis (Ph.D.)-The University of Oklahoma, 2009. 
[15] S. Meskin, Nonresidually finite one-relator groups, Trans. Amer. Math. Soc. 164 (1972) 105-114.

[16] E. Souche, Quasi-isométries et quasi-plans dans l'étude des groupes discrets, Thèse, Université de Provence, 2001.

[17] K. Whyte, The large scale geometry of the higher Baumslag-Solitar groups, Geom. Funct. Anal. 11 (2001), no. 6, 1327-1343.

[18] D. Wise, Subgroup separability of graphs of free groups with cyclic edge groups, Q. J. Math. 51 (2000), no. 1, 107-129.

Gilbert Levitt

Laboratoire de Mathématiques Nicolas Oresme

Université de Caen et CNRS (UMR 6139)

BP 5186

F-14032 Caen Cedex

France

e-mail: levitt@unicaen.fr 